\title{
TRANSITION PHENOMENA FOR LADDER EPOCHS OF RANDOM WALKS WITH SMALL NEGATIVE DRIFT
}

\author{
VITALI WACHTEL, ${ }^{*}$ University of Munich
}

\begin{abstract}
For a family of random walks $\left\{S^{(a)}\right\}$ satisfying $\mathrm{E} S_{1}^{(a)}=-a<0$, we consider ladder epochs $\tau^{(a)}=\min \left\{k \geq 1: S_{k}^{(a)}<0\right\}$. We study the asymptotic behaviour, as $a \rightarrow 0$, of $\mathrm{P}\left(\tau^{(a)}>n\right)$ in the case when $n=n(a) \rightarrow \infty$. As a consequence, we also obtain the growth rates of the moments of $\tau^{(a)}$.
\end{abstract}

Keywords: Random walk; ladder epoch; transition phenomena

2000 Mathematics Subject Classification: Primary 60G50

Secondary $60 \mathrm{G} 52$

\section{Introduction and statement of results}

\subsection{Background and purpose}

Let $X, X_{1}, X_{2}, \ldots$ be independent, identically distributed random variables. Let $S=$ $\left\{S_{n}, n \geq 0\right\}$ denote the random walk with increments $X_{i}$, that is,

$$
S_{0}:=0, \quad S_{n}:=\sum_{i=1}^{n} X_{i} .
$$

Let us first recall what is known about the first descending ladder epoch $\tau$ of $S$, i.e.

$$
\tau:=\min \left\{k \geq 1: S_{k}<0\right\} .
$$

It is well known (see, for example, [17, Theorem 17.1]) that

$$
\mathrm{P}(\tau<\infty)=1 \Longleftrightarrow \sum_{k=1}^{\infty} k^{-1} \mathrm{P}\left(S_{k}<0\right)=\infty .
$$

Under the latter condition, Rogozin [15] studied the asymptotic behaviour, as $n \rightarrow \infty$, of the tail probability $\mathrm{P}(\tau>n)$. In particular,

$$
\lim _{n \rightarrow \infty} \frac{1}{n} \sum_{k=1}^{n} \mathrm{P}\left(S_{k} \geq 0\right)=\rho \in(0,1] \quad \Longleftrightarrow \quad \mathrm{P}(\tau>n)=n^{\rho-1} \ell(n),
$$

where $\ell$ is slowly varying at infinity. Also, $\lim _{n \rightarrow \infty}(1 / n) \sum_{k=1}^{n} \mathrm{P}\left(S_{k} \geq 0\right)=0$ is equivalent to the relative stability of $\tau$. The latter means that the function $x \mapsto \int_{0}^{x} \mathrm{P}(\tau>u) \mathrm{d} u$ is slowly

Received 11 May 2009; revision received 11 October 2009.

* Postal address: Mathematical Institute, University of Munich, Theresienstrasse 39, D-80333, Munich, Germany.

Email address: wachtel@math.lmu.de 
varying at infinity. But this statement does not give any information about the asymptotic behaviour of $\mathrm{P}(\tau>n)$ in this case.

The situation when $\mathrm{E} \tau<\infty$, which is a particular case of the relative stability, was considered by Embrechts and Hawkes [5]. There it was shown that

$$
\mathrm{P}(\tau>n) \sim n^{-1} \mathrm{P}\left(S_{n}>0\right) \exp \left\{\sum_{j=1}^{\infty} j^{-1} \mathrm{P}\left(S_{j} \geq 0\right)\right\},
$$

under certain conditions on the sequence $\left\{\mathrm{P}\left(S_{n}>0\right), n \geq 1\right\}$.

If the expectation $\mathrm{E} X$ is finite then the condition $\sum_{k=1}^{\infty} k^{-1} \mathrm{P}\left(S_{k} \leq 0\right)=\infty$ is equivalent to the inequality $\mathrm{E} X \leq 0$; see again [17, Theorem 17.1]. If $\mathrm{E} X=0$ and $X$ belongs to the domain of attraction of a stable law of index $\alpha>1$, then $\lim _{n \rightarrow \infty} \mathrm{P}\left(S_{n} \geq 0\right) \in(0,1)$. (For details, see the paragraph after (11).) This yields $\lim _{n \rightarrow \infty}(1 / n) \sum_{k=1}^{n} \mathrm{P}\left(S_{k} \geq 0\right)=\rho \in(0,1)$. Then, using (2), we conclude that

$$
\mathrm{P}(\tau>n)=n^{\rho-1} \ell(n) .
$$

If $\mathrm{E} X<0$ then $\mathrm{E} \tau$ is finite; see [17, Proposition 18.1]. In this case of negative drift, Doney [4] applied the results from [5] to two special classes of random walks. He showed that if $\mathrm{E} X \in(-\infty, 0)$ and $\mathrm{P}(X>x)$ is regularly varying at $\infty$ with index $\alpha<-1$, then, as $n \rightarrow \infty$,

$$
\mathrm{P}(\tau>n) \sim \mathrm{E} \tau \mathrm{P}(X>-n \mathrm{E} X) \quad \text { as } n \rightarrow \infty .
$$

Besides this regularly varying tail case, Doney found the asymptotics of $\mathrm{P}(\tau>n)$ for random walks having negative drift and satisfying the following condition. If the equation $(\mathrm{d} / \mathrm{d} h) \mathrm{E}^{h X}=0$ has a positive solution, say $h_{0}$, then

$$
\mathrm{P}(\tau>n) \sim C\left(\frac{\mathrm{E} \mu^{\tau}-1}{\mu-1}\right) \mu^{-n} n^{-3 / 2} \quad \text { as } n \rightarrow \infty,
$$

where $\mu=1 / \mathrm{E}^{h_{0} X}$ and $C$ is a constant depending on $\mathrm{Ee}^{h X}$. The latter relation was generalised by Bertoin and Doney [2] to the case where $(\mathrm{d} / \mathrm{d} h) \mathrm{Ee}^{h X}<0$ for all $h>0$ such that $\mathrm{Ee}^{h X}<\infty$.

It should be noted that [2] and [4] are devoted to the study of the asymptotic behaviour of $\mathrm{P}\left(\tau_{x}>n\right)$ for any fixed $x \geq 0$, where $\tau_{x}:=\min \left\{k \geq 1: S_{k}<-x\right\}$. The main result can be stated as follows. If $X$ satisfies the conditions stated before (4) or (5), then there exists a function $U$ such that

$$
\lim _{n \rightarrow \infty} \frac{\mathrm{P}\left(\tau_{x}>n\right)}{\mathrm{P}(\tau>n)} \rightarrow U(x) .
$$

By studying the asymptotic behaviour, as $n \rightarrow \infty$, of $\mathrm{P}(\tau>n)$, we hope to get a good approximation for large but finite values of $n$. The quality of such an approximation depends on different parameters of the random walk. It follows from the papers mentioned above that the asymptotic behaviour of $\mathrm{P}(\tau>n)$ depends crucially on whether $\mathrm{E} X=0$ or $\mathrm{E} X<0$. Therefore, it would be very useful to clarify the influence of $\mathrm{E} X$ on $\mathrm{P}(\tau>n)$ in the case when that expectation is quite small. We illustrate the problem with the following concrete example. Let $S$ be a random walk with $\mathrm{E} X=10^{-3}$; we want to calculate the quantity $\mathrm{P}\left(\tau>10^{5}\right)$. Here we have two possibilities. On the one hand, we can say that the expectation is so small that we may apply asymptotic relations for zero-mean random walks. On the other hand, we can say that the expectation is negative and we should use (4) or (5), depending on the tail behaviour of $X$. But how do we decide which approximation is better for these values of $\mathrm{E} X$ and $n$ ? This 
question leads to the following mathematical problem. What can be said about the asymptotic behaviour of $\mathrm{P}(\tau>n)$ in the case when $\mathrm{E} X \rightarrow 0$ and $n \rightarrow \infty$ simultaneously?

In the present paper we consider this problem in the case when the random walk's increment belongs to the domain of attraction of a stable law. We shall show that there exists a function $f$ such that

(a) if $n \ll f(\mathrm{E} X)$ then we have to use (3),

(b) if $n \gg f(\mathrm{E} X)$ then we have to use formulae for random walks with negative drift,

(c) if $n \sim v f(\mathrm{E} X), v \in(0, \infty)$, then we have to use (3), but with a correction factor depending on $v$.

The last point seems to be the most interesting one. It describes transition phenomena for the ladder epoch $\tau$, which appear in the case of small drift.

Our main result, Theorem 1, is devoted to the study of this transition. There it will be clarified what the function $f$ and the correction factor look like. As a consequence, we shall obtain the claim in (a). Furthermore, Theorem 1 allows us to determine the asymptotic behaviour, as $\mathrm{E} X \rightarrow 0$, of some moments $\mathrm{E} \tau^{r}$; see Theorem 2. The expectation $\mathrm{E} \tau$ is of particular interest, since it appears in asymptotic relations connected to the claim in (b); see Theorems 3, 4, and 5.

\subsection{Transition phenomena}

We start with a more precise description of our model of random walks with asymptotically small drift. We shall consider a family of random walks $\left\{S^{(a)}, a \in\left[0, a_{0}\right]\right\}$ with drift $-a$, that is, $\mathrm{E} S_{1}^{(a)}=-a$, and investigate the asymptotic behaviour, as $a \rightarrow 0$, of the probability $\mathrm{P}\left(\tau^{(a)}>n\right)$ for $n=n(a)$, where $\tau^{(a)}$ is the first descending ladder epoch of $S^{(a)}$, as in (1).

Let $X^{(a)}$ denote a random variable that is distributed as the increments of the random walk $S^{(a)}$. It is easy to see that if $X^{(a)}$ converges in distribution, as $a \rightarrow 0$, to $X^{(0)}$ then, for every fixed $n$,

$$
\mathrm{P}\left(\tau^{(a)}>n\right) \sim \mathrm{P}\left(\tau^{(0)}>n\right) \quad \text { as } a \rightarrow 0 .
$$

A more interesting problem consists in investigating the asymptotic behaviour of the tail probability $\mathrm{P}\left(\tau^{(a)}>n\right)$ when $n=n(a) \rightarrow \infty$ as $a \rightarrow 0$. The solution to this problem depends on the structure of the family $\left\{S^{(a)}, a \in\left[0, a_{0}\right]\right\}$.

In this paper we shall assume that there exists a random variable $X$ with zero mean such that the random variables $X^{(a)}$ and $X-a$ have the same distribution for all $a \in\left[0, a_{0}\right]$. Then the random variables $S_{n}^{(a)}$ and $S_{n}^{(0)}-n a$ are equal in distribution for all $a \in\left[0, a_{0}\right]$ and $n \geq 1$. Furthermore, we restrict ourselves from now on to so-called asymptotically stable random walks. Namely, we shall always assume that the distribution of $X$ belongs to the domain of attraction of a stable law with characteristic function

$$
G_{\alpha, \beta}(t):=\exp \left\{-|t|^{\alpha}\left(1-\mathrm{i} \beta \frac{t}{|t|} \tan \frac{\pi \alpha}{2}\right)\right\}
$$

with $\alpha \in(1,2]$ and $|\beta| \leq 1$. In this case we write $X \in \mathcal{D}(\alpha, \beta)$.

Let $\left\{c_{n}, n \geq 1\right\}$ denote the sequence of positive integers specified by the relation

$$
c_{n}:=\inf \left\{u \geq 0: u^{-2} V(u) \leq n^{-1}\right\},
$$

where

$$
V(u):=\int_{-u}^{u} x^{2} \mathrm{P}(X \in \mathrm{d} x), \quad u>0 .
$$


It is known (see, for instance, [6, Chapter XVII, Section 5]) that the function $V$ is regularly varying at infinity with index $2-\alpha$ for every $X \in \mathscr{D}(\alpha, \beta)$. This implies that $\left\{c_{n}, n \geq 1\right\}$ is regularly varying with index $\alpha^{-1}$, i.e. there exists a function $l_{1}$, slowly varying at infinity, such that

$$
c_{n}=n^{1 / \alpha} l_{1}(n)
$$

In addition, the scaled sequence $\left\{S_{n}^{(0)} / c_{n}, n \geq 1\right\}$ converges in distribution, as $n \rightarrow \infty$, to the stable law corresponding to $G_{\alpha, \beta}$ in (7).

Let $\left\{Y_{\alpha, \beta}(t), t \geq 0\right\}$ denote a stable Lévy process such that $Y_{\alpha, \beta}(1)$ is distributed according to $(7)$.

It is known (see Proposition 17.5 of [17]) that the generating function of the sequence $\left\{\mathrm{P}\left(\tau^{(a)}>n\right), n \geq 0\right\}$ satisfies the identity

$$
\sum_{n=0}^{\infty} \mathrm{P}\left(\tau^{(a)}>n\right) z^{n}=\exp \left\{\sum_{n=1}^{\infty} \frac{z^{n}}{n} \mathrm{P}\left(S_{n}^{(a)} \geq 0\right)\right\}, \quad z \in(0,1) .
$$

Thus, for every $n \geq 1$, the probability $\mathrm{P}\left(\tau^{(a)}>n\right)$ is determined by $\left\{\mathrm{P}\left(S_{k}^{(a)} \geq 0\right), 1 \leq k \leq n\right\}$. From the definition of the family $S^{(a)}$ and from the asymptotic stability of $\left\{S_{n}^{(0)}, n \geq 0\right\}$, we conclude that

$$
\mathrm{P}\left(S_{n}^{(a)} \geq 0\right) \sim \mathrm{P}\left(S_{n}^{(0)} \geq 0\right) \sim \mathrm{P}\left(Y_{\alpha, \beta}(1) \geq 0\right)=: \rho
$$

for $n=n(a) \rightarrow \infty$ satisfying $n a / c_{n} \rightarrow 0$. It is known (see [18]) that

$$
\rho=\frac{1}{2}+\frac{1}{\pi \alpha} \arctan \left(\beta \tan \frac{\pi \alpha}{2}\right)
$$

for all $\alpha \in(1,2]$ and $|\beta| \leq 1$. We can easily verify that $\rho \in(0,1)$ for all $\alpha \in(1,2]$ and $|\beta| \leq 1$. Hence, we can expect that

$$
\mathrm{P}\left(\tau^{(a)}>n\right) \sim \mathrm{P}\left(\tau^{(0)}>n\right)=n^{\rho-1} \ell(n),
$$

where in the second step we have used (2). Furthermore, if $n a / c_{n} \rightarrow u \in(0, \infty)$ then

$$
\mathrm{P}\left(S_{n}^{(a)} \geq 0\right) \sim \mathrm{P}\left(Y_{\alpha, \beta}(1) \geq u\right)>0 .
$$

In this case we expect, although this conjecture is not as obvious as (12), that

$$
\mathrm{P}\left(\tau^{(a)}>n\right) \sim \mathrm{P}\left(\tau^{(0)}>n\right) G(u)
$$

for some function $G$.

The following theorem confirms conjectures (12) and (13).

Theorem 1. Suppose that $X \in \mathscr{D}(\alpha, \beta)$. If $n=n(a)$ is such that

$$
\lim _{a \rightarrow 0} \frac{a n}{c_{n}}=u \in[0, \infty)
$$

then

$$
\lim _{a \rightarrow 0} \frac{\mathrm{P}\left(\tau^{(a)}>n\right)}{\mathrm{P}\left(\tau^{(0)}>n\right)}=1-F_{\alpha, \beta}(u),
$$


where the distribution function $F_{\alpha, \beta}$ can be described by the equality

$$
\begin{aligned}
\int_{0}^{\infty} & \mathrm{e}^{-\lambda x} x^{\rho-1}\left(1-F_{\alpha, \beta}\left(x^{1-1 / \alpha}\right)\right) \mathrm{d} x \\
& =C \exp \left\{-\int_{0}^{\infty} \frac{1-\mathrm{e}^{-\lambda t}}{t} \mathrm{P}\left(Y_{\alpha, \beta}(t)-t>0\right) \mathrm{d} t\right\}, \quad \lambda \geq 0
\end{aligned}
$$

with $\rho$ defined as in (11) and $C$ specified by the condition $F_{\alpha, \beta}(0)=0$.

The existence of the limit in (15) is an easy consequence of the invariance principle for random walks conditioned to stay positive, which was proved in [3]. The most difficult part of the proof is the derivation of characterisation (16) of the limiting distribution $F_{\alpha, \beta}$; see Section 3.

It follows from (9) that (14) is equivalent to

$$
n \sim u^{\alpha /(\alpha-1)}\left(\frac{1}{a}\right)^{\alpha /(\alpha-1)} l^{*}\left(\frac{1}{a}\right) \quad \text { as } a \rightarrow 0,
$$

where $l^{*}$ is slowly varying at infinity, which is determined by $l_{1}$. Therefore, the statement of Theorem 1 can be reformulated as follows. If $n=n(a)$ satisfies

$$
n \sim v\left(\frac{1}{a}\right)^{\alpha /(\alpha-1)} l^{*}\left(\frac{1}{a}\right) \quad \text { as } a \rightarrow 0
$$

for some $v \geq 0$ then

$$
\lim _{a \rightarrow 0} \frac{\mathrm{P}\left(\tau^{(a)}>n\right)}{\mathrm{P}\left(\tau^{(0)}>n\right)}=1-F_{\alpha, \beta}\left(v^{1-1 / \alpha}\right) .
$$

In particular, if (17) holds with $v=0$ then $\mathrm{P}\left(\tau^{(a)}>n\right) \sim \mathrm{P}\left(\tau^{(0)}>n\right)$. Roughly speaking, (3) gives a rather good approximation in the case when $n$ is much smaller than $(1 / a)^{\alpha /(\alpha-1)} l^{*}(1 / a)$. But if $(1 / a)^{\alpha /(\alpha-1)} l^{*}(1 / a)$ and $n$ are comparable, then we have to use a correction factor, given by the right-hand side of (18). To calculate this correction for concrete values of $v$, we need to know the form of the distribution function $F_{\alpha, \beta}$. We are able to give an explicit expression for $F_{\alpha, \beta}$ only in some special cases. We shall see in the proof of Theorem 1 that

$$
1-F_{\alpha, \beta}(u)=\mathrm{P}\left(\inf _{t \leq 1}\left(M_{\alpha, \beta}(t)-u t\right) \geq 0\right),
$$

where $\left\{M_{\alpha, \beta}(t), t \in[0,1]\right\}$ is the meander of $Y_{\alpha, \beta}$. Using the construction of the meander via the limit of conditioned distributions of the original process $Y_{\alpha, \beta}$, we shall show that

$$
1-F_{2,0}(u)=u \int_{u}^{\infty} v^{-2} \mathrm{e}^{-v^{2} / 2} \mathrm{~d} v
$$

and

$$
1-F_{\alpha, 1}(u)=\frac{u^{1 /(\alpha-1)}}{(\alpha-1) g_{\alpha, 1}(0)} \int_{u}^{\infty} v^{-\alpha /(\alpha-1)} g_{\alpha, 1}(v) \mathrm{d} v, \quad \alpha \in(1,2),
$$

where $g_{\alpha, \beta}$ denotes the density function of the random variable $Y_{\alpha, \beta}(1)$. For all other values of $\alpha$ and $\beta$, the explicit form of $F_{\alpha, \beta}$ remains unknown. 
Remark 1. The expression on the right-hand side of (16) is known (see [1, p. 168]) to be the Laplace transform of the random variable

$$
T_{\max }:=\sup \left\{t>0: Y_{\alpha, \beta}(t)-t=\max _{u \geq 0}\left(Y_{\alpha, \beta}(u)-u\right)\right\} .
$$

Let $f_{\max }$ denote the density function of this random variable. Then from (16) we can obtain the equality

$$
1-F_{\alpha, \beta}(x)=C x^{\alpha(1-\rho) /(\alpha-1)} f_{\max }\left(x^{\alpha /(\alpha-1)}\right), \quad x>0 .
$$

Having this relation we can obtain the explicit form of $f_{\max }$ in the case of Brownian motion $(\alpha=2$ and $\beta=0)$ and in the case of spectrally positive Lévy processes $(\alpha \in(1,2)$ and $\beta=1)$.

We now turn our attention to the moments of $\tau^{(a)}$.

It was shown by Gut [7] that the condition $\mathrm{E}(\max \{0, X\})^{r}<\infty$ for some $r>0$ is necessary and sufficient for the finiteness of $\mathrm{E}\left(\tau^{(a)}\right)^{r}$. Therefore, the condition $X \in \mathscr{D}(\alpha, \beta)$ yields the finiteness of $\mathrm{E}\left(\tau^{(a)}\right)^{r}$ for all $r<\alpha$.

From the bound

$$
\mathrm{P}\left(\tau^{(a)}>n\right) \leq \mathrm{P}\left(\tau^{(0)}>n\right) \text { for all } n \geq 0
$$

and (6), using dominated convergence, we infer that

$$
\lim _{a \rightarrow 0} \mathrm{E}\left(\tau^{(a)}\right)^{r}=\mathrm{E}\left(\tau^{(0)}\right)^{r}<\infty
$$

for all $r \in(0,1-\rho)$. Furthermore, it easily follows from Theorem 1 and (12) that

$$
\lim _{a \rightarrow 0} \mathrm{E}\left(\tau^{(a)}\right)^{r}=\infty \quad \text { for all } r>1-\rho .
$$

Theorem 1 allows us to determine the rate of growth as $a \rightarrow 0$ of $\mathrm{E}\left(\tau^{(a)}\right)^{r}$ for $r \in(1-\rho, \alpha)$.

Theorem 2. Suppose that $X \in \mathscr{D}(\alpha, \beta)$. Then, for every $r \in(1-\rho, \alpha)$, there exists a function $L_{r}$ slowly varying at infinity such that

$$
\mathrm{E}\left(\tau^{(a)}\right)^{r}=L_{r}\left(\frac{1}{a}\right) a^{-\alpha(r+\rho-1) /(\alpha-1)},
$$

with $\rho$ defined as in (11).

This is already known in some particular cases, which we now mention.

First of all we note that if the second moment of $X$ is finite then, applying dominated convergence, we can show that $S_{\tau^{(a)}}^{(a)} \rightarrow \operatorname{E~} S_{\tau^{(0)}}^{(0)}$ as $a \rightarrow 0$. Thus, using the Wald identity and the well-known equality (see [17, Proposition 18.5])

$$
-\mathrm{E} S_{\tau^{(0)}}^{(0)}=\frac{\left(\mathrm{E} X^{2}\right)^{1 / 2}}{\sqrt{2}} \exp \left\{\sum_{k=1}^{\infty} k^{-1}\left(\mathrm{P}\left(S_{k}^{(0)} \geq 0\right)-\frac{1}{2}\right)\right\}
$$

we obtain, as $a \rightarrow 0$,

$$
\mathrm{E} \tau^{(a)} \sim \frac{-\mathrm{E} S_{\tau^{(0)}}^{(0)}}{a}=\frac{\left(\mathrm{E} X^{2}\right)^{1 / 2}}{a \sqrt{2}} \exp \left\{\sum_{k=1}^{\infty} k^{-1}\left(\mathrm{P}\left(S_{k}^{(0)} \geq 0\right)-\frac{1}{2}\right)\right\} .
$$


Furthermore, the asymptotic behaviour of $\mathrm{E} \tau^{(a)}$ in the case of a non-Gaussian stable limit, that is, $\alpha<2$, was recently studied by Lotov [9]. He proved that

$$
\mathrm{E} \tau^{(a)}=a^{-\alpha \rho /(\alpha-1)+o(1)} \quad \text { as } a \downarrow 0
$$

in this case. Moreover, he showed that (19) with $r=1$ holds under the additional condition that

$$
\sum_{k=1}^{\infty} \frac{1}{k} \sup _{x \in \mathbb{R}}\left|\mathrm{P}\left(S_{k}^{(0)}>c_{k} x\right)-\mathrm{P}\left(Y_{\alpha, \beta}>x\right)\right|<\infty .
$$

Having expressions for the expectation $\mathrm{E} \tau^{(a)}$ we can describe the asymptotic behaviour of some further characteristics of the random walk $\left\{S_{n}^{(a)}, n \geq 0\right\}$. First, from the Wald identity and Theorem 2, we obtain the equality

$$
\mathrm{E} S_{\tau^{(a)}}^{(a)}=-a \mathrm{E} \tau^{(a)}=-L_{1}\left(\frac{1}{a}\right) a^{1-\alpha \rho /(\alpha-1)} .
$$

Second, it is well known that the stopping time $\tau_{+}^{(a)}:=\min \left\{k \geq 1: S_{k}^{(a)} \geq 0\right\}$ is infinite with positive probability and that $\mathrm{P}\left(\tau_{+}^{(a)}=\infty\right)=1 / \mathrm{E} \tau^{(a)}$. Then, using Theorem 2 once again, we obtain

$$
\mathrm{P}\left(\tau_{+}^{(a)}=\infty\right)=\frac{a^{\alpha \rho /(\alpha-1)}}{L_{1}(1 / a)} .
$$

To conclude this subsection, we note that our assumption that the distributions of $X^{(a)}$ and $X-a$ are equal can be weakened. First of all we note that if $X^{(a)}$ satisfies the conditions

$$
\mathrm{E} X^{(a)}=-a \text { and } \lim _{a \rightarrow 0} \mathrm{E}\left(X^{(a)}\right)^{2}=\sigma^{2} \in(0, \infty),
$$

then the results of the present subsection still hold. Moreover, in the case of an infinite second moment, the results of the present subsection remain valid if $X^{(a)}=X-a+Y^{(a)}$ in distribution, where $X \in \mathscr{D}(\alpha, \beta)$ for some $\alpha \in(1,2)$ and $Y^{(a)}$ is such that

$$
\mathrm{E} Y^{(a)}=0, \quad Y^{(a)} \rightarrow 0 \text { in law and } \sup _{a \in\left[0, a_{0}\right]} \mathrm{E}\left|Y^{(a)}\right|^{\alpha+\delta}<\infty \text { for some } \delta>0 .
$$

We did not use these generalisations in the statements of our theorems because of results in the next subsection, where we need the assumption that $X^{(a)}=X-a$ in law.

\subsection{Results on large deviations}

If $n a / c_{n} \rightarrow \infty$ then Theorem 1 says only that

$$
\mathrm{P}\left(\tau^{(a)}>n\right)=o\left(\mathrm{P}\left(\tau^{(0)}>n\right)\right) \quad \text { as } a \rightarrow 0 .
$$

Our next purpose is to refine this relation and to find the rate of divergence of $\mathrm{P}\left(\tau^{(a)}>n\right)$ in the abovementioned domain of large deviations for $\tau^{(a)}$. To proceed in this situation, we need to know the asymptotic behaviour of $\mathrm{P}\left(S_{n}^{(a)}>0\right)$ for $n a / c_{n} \rightarrow \infty$. It follows from the definition of $S_{n}^{(a)}$ that $\mathrm{P}\left(S_{n}^{(a)}>0\right)=\mathrm{P}\left(S_{n}^{(0)}>n a\right)$. Thus, the assumption that $n a / c_{n} \rightarrow \infty$ means that we are in the domain of large deviations for $S_{n}^{(0)}$. Since the behaviour of large deviation probabilities depends crucially on whether the limit of $S_{n}^{(0)} / c_{n}$ is Gaussian or strictly stable, i.e. $\alpha \in(1,2)$, we consider these two cases separately. 
If $S_{n}^{(0)}$ belongs to the domain of attraction of a strictly stable law then, as is well known,

$$
\mathrm{P}\left(S_{n}^{(0)} \geq x_{n}\right) \sim n \mathrm{P}\left(X \geq x_{n}\right)
$$

for any sequence $x_{n}$ satisfying $x_{n} / c_{n} \rightarrow \infty$. This relation allows us to obtain the following result.

Theorem 3. Suppose that $X \in \mathcal{D}(\alpha, \beta)$ for some $1<\alpha<2$ and $\beta>-1$. If $n=n(a)$ is such that $n a / c_{n} \rightarrow \infty$ then

$$
\mathrm{P}\left(\tau^{(a)}>n\right) \sim \mathrm{E} \tau^{(a)} \mathrm{P}(X \geq n a) \text { as } a \rightarrow 0 .
$$

The right-hand side of (20) coincides with that of (4). Roughly speaking, if $n$ is very large then the asymptotic behaviour of $\mathrm{P}\left(\tau^{(a)}>n\right)$ is as in the case of the fixed negative drift. But there is one crucial difference between fixed and asymptotically small drift: the expectation $\mathrm{E} \tau^{(a)}$ grows unbounded if $a \rightarrow 0$, and is a constant when the drift is fixed. Therefore, (20) would be useless without Theorem 2 .

We turn our attention to the case when $\sigma^{2}:=\mathrm{E} X^{2}$ is finite. Here we shall assume without loss of generality that $\sigma^{2}=1$. Under this condition, we have $c_{n}=\sqrt{n}$. Then the condition that $a n / c_{n} \rightarrow \infty$ reads as $n a^{2} \rightarrow \infty$. In this case of finite variance the asymptotic behaviour of $\mathrm{P}\left(S_{n}^{(0)}>x_{n}\right)$ depends not only on the tail behaviour of $X$, but also on the rate of the growth of $x_{n}$. If $x_{n}$ does not grow very fast $\left(x_{n}=o\left(r_{1}(n)\right)\right.$ for some $r_{1}(n)$ depending on the distribution of $X)$ then we have an asymptotic expression for $\mathrm{P}\left(S_{n}^{(0)}>x_{n}\right)$ in terms of the so-called Cramér series (for the definition of the Cramér series see, for example, [14, Chapter VIII]). For this type of large deviation, we have the following result.

Theorem 4. Assume that $\mathrm{E} X^{2}=1, n=n(a)$ is such that $n a^{2} \rightarrow \infty$, and that

$$
\mathrm{P}\left(S_{j}^{(0)} \geq j a\right) \sim \bar{\Phi}(\sqrt{j} a) \exp \left\{j a^{3} \lambda_{m}(a)\right\} \text { uniformly in } j \in\left[a^{-2}, n\right],
$$

where $\lambda_{m}(u)$ is the partial sum in the Cramér series containing the first $m$ terms and $\bar{\Phi}(x):=$ $\int_{x}^{\infty}(1 / \sqrt{2 \pi}) \mathrm{e}^{-u^{2} / 2} \mathrm{~d} u$. Then

$$
\mathrm{P}\left(\tau^{(a)}>n\right) \sim 2 \mathrm{E} \tau^{(a)} \frac{1}{n} \bar{\Phi}(\sqrt{n} a) \exp \left\{n a^{3} \lambda_{m}(a)\right\}
$$

Condition (21) has one essential disadvantage: it involves the whole sequence $\left\{S_{k}^{(0)}, k \geq 0\right\}$. We now list some restrictions on the distribution of $X$, which imply the validity of (21).

Nagaev [11] proved that the condition $\mathrm{E}|X|^{k}<\infty$ with some $k>2$ implies that the relation

$$
\mathrm{P}\left(S_{n}^{(0)} \geq x\right) \sim \bar{\Phi}\left(\frac{x}{\sqrt{n}}\right) \quad \text { as } n \rightarrow \infty
$$

holds uniformly in $x \leq \sqrt{(k / 2-1) n \log n}$. Thus, the existence of $\mathrm{E}|X|^{k}$ for some $k>2$ yields (21) with $m=0$ for all $n$ satisfying

$$
n \leq\left(\frac{1}{2} k-1\right) a^{-2} \log a^{-2} .
$$

Furthermore, it has been proved by Nagaev [10] and Rozovskii [16] that if $\mathrm{P}(X>x)$ is regularly varying at infinity with index $p<-2$ then, under some additional restrictions on the left tail,

$$
\mathrm{P}\left(S_{n}^{(0)} \geq x\right) \sim \bar{\Phi}\left(\frac{x}{\sqrt{n}}\right)+n \mathrm{P}(X>x+\sqrt{n}) \quad \text { uniformly on } x>0 .
$$


Thus, (22) holds for all $x \leq C \sqrt{n \log n}$ for any $C<(p-2)^{1 / 2}$. Consequently, (21) with $m=0$ holds for

$$
n \leq C a^{-2} \log a^{-2}, \quad C<(p-2)^{1 / 2} .
$$

Osipov [13] found necessary and sufficient conditions under which the relation

$$
\mathrm{P}\left(S_{n}^{(0)} \geq x\right) \sim \bar{\Phi}\left(\frac{x}{\sqrt{n}}\right) \exp \left\{\frac{x^{3}}{n^{2}} \lambda_{[1 /(1-\gamma)]}\left(\frac{x}{n}\right)\right\}
$$

holds uniformly in $0 \leq x \leq n^{\gamma}, \frac{1}{2}<\gamma<1$, where [t] denotes the integer part of $t$. If these conditions are fulfilled then, obviously, (21) holds with $m=[1 /(1-\gamma)]$ for all $n \leq a^{1 /(1-\gamma)}$.

It is well known that if $X$ satisfies the Cramér condition $\left(\mathrm{E}^{h|X|}<\infty\right.$ for some $\left.h>0\right)$ then (21) holds with $m=\infty$ and for all $n$ satisfying $n a^{2} \rightarrow \infty$. Thus, Theorems 1 and 4 describe the behaviour of $\mathrm{P}\left(\tau^{(a)}>n\right)$ for any choice of $n=n(a)$ and any random walk satisfying the Cramér condition.

It is easy to see that the statement of Theorem 4 can be rewritten as follows. If (21) holds then

$$
\mathrm{P}\left(\tau^{(a)}>n\right) \sim \frac{2}{\sqrt{2 \pi}} a^{-1} \mathrm{E} \tau^{(a)} n^{-3 / 2} \mathrm{e}^{-n \xi(a)},
$$

where

$$
\xi(a):=\frac{a^{2}}{2}-a^{3} \lambda_{m}(a) .
$$

Furthermore, in the proof of Theorem 4 we shall see that

$$
\frac{\mathrm{E}\left(\mathrm{e}^{\xi(a) \tau^{(a)}}, \tau^{(a)} \leq n\right)-1}{\mathrm{e}^{\xi(a)}-1} \sim 2 \mathrm{E} \tau^{(a)} .
$$

Thus,

$$
\mathrm{P}\left(\tau^{(a)}>n\right) \sim \frac{1}{a \sqrt{2 \pi}} \frac{\mathrm{E}\left(\mathrm{e}^{\xi(a) \tau^{(a)}}, \tau^{(a)} \leq n\right)-1}{\mathrm{e}^{\xi(a)}-1} n^{-3 / 2} \mathrm{e}^{-n \xi(a)},
$$

which is rather close to relation (5). If, additionally, $X$ satisfies the Cramér condition, implying (21) with $m=\infty$, then we can replace the truncated expectation $\mathrm{E}\left(\mathrm{e}^{\xi(a) \tau^{(a)}}, \tau^{(a)} \leq n\right)$ by $\mathrm{E}\left(\mathrm{e}^{\xi(a) \tau^{(a)}}\right)$ :

$$
\mathrm{P}\left(\tau^{(a)}>n\right) \sim \frac{1}{a \sqrt{2 \pi}} \frac{\mathrm{E}\left(\mathrm{e}^{\xi(a) \tau^{(a)}}\right)-1}{\mathrm{e}^{\xi(a)}-1} n^{-3 / 2} \mathrm{e}^{-n \xi(a)} .
$$

It follows from the definition of the Cramér series that $\xi(a)$, defined in (24), is the unique positive solution to the equation $(\mathrm{d} / \mathrm{d} h) \mathrm{E}^{h X^{(a)}}=0$. Therefore, (25) is an analog of (5) for random walks with vanishing drift.

Another type of large deviation behaviour appears in the case when $x_{n}$ grows fast, i.e. $x_{n} \gg r_{2}(n)$ and the tail of $X$ varies in an appropriate way. (Recall that $a_{n} \gg b_{n}$ means that $a_{n} / b_{n} \rightarrow \infty$.) Here, as in the case of the non-Gaussian stable limit, we have $\mathrm{P}\left(S_{n}^{(0)} \geq x_{n}\right) \sim n \mathrm{P}\left(X \geq x_{n}\right)$. We consider only the case when the tail of $X$ is regularly varying.

Theorem 5. Assume that $\mathrm{P}(X \geq x)$ is regularly varying at infinity with index $p<-2$ and

$$
\int_{|x|>y} x^{2} \mathrm{P}(X \in \mathrm{d} x)=o\left(\frac{1}{\log y}\right) \text { as } y \rightarrow \infty .
$$


Then, as $a \rightarrow 0$,

$$
\mathrm{P}\left(\tau^{(a)}>n\right) \sim \mathrm{E} \tau^{(a)} \mathrm{P}(X \geq n a)
$$

for any $n=n(a)$ satisfying the inequality $n(a) \geq C a^{-2} \log a^{-2}$ with some $C>(p-2)^{1 / 2}$.

After Theorem 4 we mentioned that, in the case of regularly varying tails, (21) holds for all $n \leq C a^{-2} \log a^{-2}, C<(p-2)^{1 / 2}$. Therefore, the behaviour of $\mathrm{P}\left(\tau^{(a)}>n\right)$ remains unclear only for $n$ satisfying $\left(n a^{2} / \log a^{-2}\right) \rightarrow(p-2)^{1 / 2}$. We conjecture that if the conditions of Theorem 5 hold then, in agreement with (23),

$$
\mathrm{P}\left(\tau^{(a)}>n\right) \sim 2 \mathrm{E} \tau^{(a)} \frac{1}{n} \bar{\Phi}(\sqrt{n} a)+\mathrm{E} \tau^{(a)} \mathrm{P}(X \geq \sqrt{n}+n a)
$$

for all $n$ satisfying $n a^{2} \rightarrow \infty$.

The remaining part of the paper is organised as follows. In the next section we derive an upper bound for the probability $\mathrm{P}\left(\tau^{(a)}>n\right)$, which is crucial for the proof of Theorem 2 . This proof will be given in Section 4. Section 3 is devoted to the proof of Theorem 1. Finally, Theorems 3, 4, and 5 will be proved in Section 4.

\section{Upper bounds for the tail of $\tau^{(a)}$}

It follows from (10) that in order to obtain upper bounds for $\mathrm{P}\left(\tau^{(a)}>n\right)$ we need inequalities for $\mathrm{P}\left(S_{n}^{(a)} \geq 0\right)=\mathrm{P}\left(S_{n}^{(0)} \geq n a\right)$. In the following lemma we adapt one of the well-known FukNagaev inequalities for our purposes.

Lemma 1. Assume that $X \in \mathscr{D}(\alpha, \beta)$. Then there exists a constant $C$ such that the inequality

$$
\mathrm{P}\left(S_{n}^{(0)} \geq x\right) \leq n \mathrm{P}\left(X \geq \frac{x}{3}\right)+C\left(\frac{n V(x)}{x^{2}}\right)^{2}
$$

holds for all $x>0$ and $n \geq 1$.

Proof. Applying Theorem 1.2 of [12] with $t=2$ we have

$$
\mathrm{P}\left(S_{n}^{(0)} \geq x\right) \leq n \mathrm{P}(X \geq y)+\mathrm{e}^{x / y}\left(\frac{n V(y)}{x y}\right)^{x / y+n V(y) / y^{2}-n \mu(y) / y},
$$

where $\mu(y):=\mathrm{E}(X,|X| \leq y)$.

Since $\mathrm{E} X=0$,

$$
|\mu(y)|=\left|\int_{|x|>y} x \mathrm{P}(X \in \mathrm{d} x)\right| \leq \int_{x>y} x \mathrm{P}(|X| \in \mathrm{d} x)=y \mathrm{P}(|X|>y)+\int_{y}^{\infty} \mathrm{P}(|X|>x) \mathrm{d} x .
$$

It is well known that the assumption $X \in \mathscr{D}(\alpha, \beta)$ yields

$$
\lim _{x \rightarrow \infty} \frac{x^{2} \mathrm{P}(|X|>x)}{V(x)}=\frac{2-\alpha}{\alpha} .
$$

Therefore, as $y \rightarrow \infty$,

$$
|\mu(y)| \leq\left(\frac{2-\alpha}{\alpha}+o(1)\right)\left(\frac{V(y)}{y}+\int_{y}^{\infty} \frac{V(x)}{x^{2}} \mathrm{~d} x\right)=\left(\frac{2-\alpha}{\alpha-1}+o(1)\right) \frac{V(y)}{y} .
$$


In the last step we used the relation

$$
\int_{y}^{\infty} \frac{V(x)}{x^{2}} \mathrm{~d} x \sim \frac{1}{\alpha-1} \frac{V(y)}{y} \quad \text { as } y \rightarrow \infty,
$$

which follows from the fact that $V(x)$ is regularly varying with index $2-\alpha$. As a result, we have the bound

$$
\frac{V(y)}{y^{2}}-\frac{\mu(y)}{y} \geq\left(\frac{2 \alpha-3}{\alpha-1}+o(1)\right) \frac{V(y)}{y^{2}} .
$$

It follows from definition (8) of the sequence $\left\{c_{n}\right\}$ that $V\left(c_{n}\right) / c_{n}^{2} \sim n^{-1}$ as $n \rightarrow \infty$. Consequently, there exists a constant $C(\alpha)$ such that

$$
\frac{V(y)}{y^{2}}-\frac{\mu(y)}{y} \geq-\frac{1}{n}
$$

for all $y>C(\alpha) c_{n}$. From this bound and (27) with $y=x / 3$ we obtain

$$
\mathrm{P}\left(S_{n}^{(0)} \geq x\right) \leq n \mathrm{P}\left(X \geq \frac{x}{3}\right)+27 \mathrm{e}^{3}\left(\frac{n V(y)}{x^{2}}\right)^{2}, \quad x \geq 3 C(\alpha) c_{n} .
$$

This inequality, together with monotonicity of $V$, implies that the desired result holds for $x>C(\alpha) c_{n}$. Noting that

$$
\min _{n \geq 1} \inf _{x \leq 3 C(\alpha) c_{n}} \frac{n V(x)}{x^{2}}>0,
$$

we complete the proof of the lemma.

In order to 'translate' bounds for $\mathrm{P}\left(S_{n}^{(0)}>n a\right)$ into bounds for $\mathrm{P}\left(\tau^{(a)}>n\right)$, we shall use the recurrent relation

$$
n \mathrm{P}\left(\tau^{(a)}>n\right)=\sum_{j=0}^{n-1} \mathrm{P}\left(\tau^{(a)}>j\right) \mathrm{P}\left(S_{n-j}^{(0)}>(n-j) a\right),
$$

which can be obtained by differentiating (10).

Proposition 1. The inequality $\mathrm{P}\left(\tau^{(a)}>n\right) \leq C \mathrm{E} \tau^{(a)} V(n a) /(n a)^{2}$ is valid for all $a>0$ and all $n \geq n_{a}:=\min \left\{n \geq 1:\right.$ an $\left.>c_{n}\right\}$.

Proof. Using Lemma 1, we have

$$
\begin{aligned}
& \sum_{0 \leq j<n / 2} \mathrm{P}\left(\tau^{(a)}>j\right) \mathrm{P}\left(S_{n-j}^{(0)}>(n-j) a\right) \\
& \quad \leq \sum_{0 \leq j<n / 2} \mathrm{P}\left(\tau^{(a)}>j\right)\left((n-j) \mathrm{P}\left(X \geq \frac{(n-j) a}{3}\right)+C\left(\frac{(n-j) V((n-j) a)}{((n-j) a)^{2}}\right)^{2}\right) \\
& \quad \leq\left(n \mathrm{P}\left(X \geq \frac{n a}{6}\right)+C\left(\frac{n V(n a)}{(n a)^{2}}\right)^{2}\right) \sum_{0 \leq j<n / 2} \mathrm{P}\left(\tau^{(a)}>j\right) \\
& \quad \leq \mathrm{E} \tau^{(a)}\left(n \mathrm{P}\left(X \geq \frac{n a}{6}\right)+C\left(\frac{n V(n a)}{(n a)^{2}}\right)^{2}\right) \\
& \quad \leq n \mathrm{E} \tau^{(a)}\left(\mathrm{P}\left(X \geq \frac{n a}{6}\right)+C \frac{V(n a)}{(n a)^{2}}\right) .
\end{aligned}
$$


In the last step we used definition (8) of $c_{n}$ and the bound $a n \geq c_{n}$, which follows from the assumption that $n \geq n_{a}$.

Furthermore, using the Markov inequality, we obtain

$$
\sum_{n / 2 \leq j \leq n-1} \mathrm{P}\left(\tau^{(a)}>j\right) \mathrm{P}\left(S_{n-j}^{(0)}>(n-j) a\right) \leq \frac{2 \mathrm{E} \tau^{(a)}}{n} \sum_{k=1}^{n} \mathrm{P}\left(S_{k}^{(0)} \geq k a\right) .
$$

Applying Lemma 1, we obtain

$$
\begin{aligned}
\sum_{k=1}^{n} \mathrm{P}\left(S_{k}^{(0)} \geq k a\right) & \leq n_{a}+\sum_{k=n_{a}}^{n} \mathrm{P}\left(S_{k}^{(0)} \geq k a\right) \\
& \leq n_{a}+\sum_{k=n_{a}}^{n} k \mathrm{P}\left(X \geq \frac{k a}{3}\right)+C \sum_{k=n_{a}}^{n} \frac{V^{2}(k a)}{k^{2} a^{4}}
\end{aligned}
$$

Since $V(x)$ is regularly varying with index $2-\alpha$,

$$
\begin{aligned}
\sum_{k=n_{a}}^{n} \frac{V^{2}(k a)}{k^{2} a^{4}} & \leq C a^{-2} \sum_{k=n_{a}}^{n} \frac{V^{2}(k a)}{(k a)^{2}} \\
& \leq C a^{-3} \int_{a n_{a}}^{a n} \frac{V^{2}(x)}{x^{2}} \mathrm{~d} x \\
& \leq C a^{-3} V(\text { an }) \int_{a n_{a}}^{a n} \frac{V(x)}{x^{2}} \mathrm{~d} x \\
& \leq C a^{-3} V(\text { an }) \frac{V\left(a n_{a}\right)}{a n_{a}} .
\end{aligned}
$$

From the definitions of $c_{n}$ and $n_{a}$, we infer that

$$
V\left(a n_{a}\right) \sim V\left(c_{n_{a}}\right) \sim \frac{c_{n_{a}}^{2}}{n_{a}} \sim a^{2} n_{a} .
$$

Applying this relation to the last line in (33), we obtain the bound

$$
\sum_{k=n_{a}}^{n} \frac{V^{2}(k a)}{k^{2} a^{4}} \leq C a^{-2} V(a n)
$$

Furthermore,

$$
\begin{aligned}
\sum_{k=n_{a}}^{n} k \mathrm{P}\left(X \geq \frac{k a}{3}\right) & \leq C a^{-2} \int_{a n_{a}}^{a n} x \mathrm{P}\left(X>\frac{x}{3}\right) \mathrm{d} x \\
& \leq a^{-2} \int_{0}^{n a} x \mathrm{P}(|X|>x) \mathrm{d} x \\
& =\frac{a^{-2}}{2}\left(V(a n)+(a n)^{2} \mathrm{P}(|X|>a n)\right)
\end{aligned}
$$

where in the last step we used integration by parts. Combining (32), (35), and (36), we have

$$
\sum_{k=1}^{n} \mathrm{P}\left(S_{k}^{(0)} \geq k a\right) \leq C n_{a}+C a^{-2} V(a n)+n^{2} \mathrm{P}(|X|>a n) .
$$


It is easy to see that (34) yields $n_{a} \sim a^{-2} V\left(a n_{a}\right)$. From this relation and monotonicity of $V(x)$, we conclude that $n_{a} \leq C a^{-2} V(a n)$ for all $n \geq n_{a}$. Applying this bound to (37) we obtain

$$
\sum_{k=1}^{n} \mathrm{P}\left(S_{k}^{(0)} \geq k a\right) \leq C a^{-2} V(a n)+n^{2} \mathrm{P}(|X|>a n) .
$$

Combining (30), (31), and (38), we arrive at the inequality

$$
\sum_{j=0}^{n} \mathrm{P}\left(\tau^{(a)}>j\right) \mathrm{P}\left(S_{n-j}^{(0)}>(n-j) a\right) \leq C n \mathrm{E} \tau^{(a)}\left(\mathrm{P}\left(|X| \geq \frac{n a}{6}\right)+\frac{V(n a)}{(n a)^{2}}\right) .
$$

It follows from (28) that

$$
\mathrm{P}(|X|>x) \leq C \frac{V(x)}{x^{2}} .
$$

Therefore, the right-hand side of (39) is bounded by $C n \mathrm{E} \tau^{(a)} V(n a) /(n a)^{2}$. Thus, the statement of the proposition follows from (29).

\section{Proof of Theorem 1}

From the definition of the first ladder epoch $\tau^{(a)}$ we obtain

$$
\begin{aligned}
\mathrm{P}\left(\tau^{(a)}>n\right) & =\mathrm{P}\left(\min _{1 \leq k \leq n}\left(S_{k}^{(0)}-k a\right)>0\right) \\
& =\mathrm{P}\left(\min _{1 \leq k \leq n} S_{k}^{(0)}>0\right) \mathrm{P}\left(\min _{1 \leq k \leq n}\left(S_{k}^{(0)}-k a\right)>0 \mid \min _{1 \leq k \leq n} S_{k}^{(0)}>0\right) \\
& =\mathrm{P}\left(\tau^{(0)}>n\right) \mathrm{P}\left(\min _{1 \leq k \leq n}\left(\frac{S_{k}^{(0)}}{c_{n}}-\frac{k}{n} \frac{a n}{c_{n}}\right)>0 \mid \min _{1 \leq k \leq n} S_{k}^{(0)}>0\right) .
\end{aligned}
$$

Doney [3] showed that $\left\{S_{\left[t c_{n}\right]}^{(0)} / c_{n}, t \in[0,1] \mid \min _{1 \leq k \leq n} S_{k}^{(0)}>0\right\}$ converges weakly, as $n \rightarrow \infty$, to the Lévy meander $\left\{M_{\alpha, \beta}(t), t \in[0,1]\right\}$. This yields

$$
\begin{aligned}
\lim _{n \rightarrow \infty} \mathrm{P}\left(\min _{1 \leq k \leq n}\left(\frac{S_{k}^{(0)}}{c_{n}}-\frac{k}{n} \frac{a n}{c_{n}}\right)>0 \mid \min _{1 \leq k \leq n} S_{k}^{(0)}>0\right) & =\mathrm{P}\left(\min _{0 \leq t \leq 1}\left(M_{\alpha, \beta}(t)-u t\right)>0\right) \\
& =: 1-F_{\alpha, \beta}(u)
\end{aligned}
$$

It is obvious that $F_{\alpha, \beta}(u)$ is monotonously increasing and $\lim _{u \rightarrow \infty} F_{\alpha, \beta}(u)=1$.

It is known that the corresponding meander $M_{\alpha, \beta}$ can be defined as a weak limit, as $\varepsilon \rightarrow 0$, of $Y_{\alpha, \beta}$ starting from $\varepsilon>0$ and conditioned to stay positive up to time 1:

$$
\begin{aligned}
& \mathcal{L}\left\{M_{\alpha, \beta}(t), t \in[0,1]\right\} \\
& \quad=\lim _{\varepsilon \rightarrow 0} \mathcal{L}\left\{Y_{\alpha, \beta}(t), t \in[0,1] \mid \inf _{0 \leq t \leq 1} Y_{\alpha, \beta}(t)>0, Y_{\alpha, \beta}(0)=\varepsilon\right\} .
\end{aligned}
$$

Therefore,

$$
1-F_{\alpha, \beta}(u)=\lim _{\varepsilon \rightarrow 0} \frac{\mathrm{P}\left(\inf _{0 \leq t \leq 1}\left(Y_{\alpha, \beta}(t)-u t\right)>0 \mid Y_{\alpha, \beta}(0)=\varepsilon\right)}{\mathrm{P}\left(\inf _{0 \leq t \leq 1} Y_{\alpha, \beta}(t)>0 \mid Y_{\alpha, \beta}(0)=\varepsilon\right)} .
$$


Define $H_{\alpha, \beta}^{(u)}(z):=\min \left\{t: Y_{\alpha, \beta}(t)-u t \leq z \mid Y_{\alpha, \beta}(0)=0\right\}$. Then

$$
1-F_{\alpha, \beta}(u)=\lim _{\varepsilon \rightarrow 0} \frac{\mathrm{P}\left(H_{\alpha, \beta}^{(u)}(-\varepsilon)>1\right)}{\mathrm{P}\left(H_{\alpha, \beta}^{(0)}(-\varepsilon)>1\right)} .
$$

In the case of the Brownian motion, that is, $\alpha=2$ and $\beta=0$, we can calculate the limit explicitly. Indeed, it is known that $H_{2,0}^{(u)}(-\varepsilon)$ has the density

$$
\frac{\varepsilon}{\sqrt{2 \pi} t^{3 / 2}} \exp \left\{-\frac{(u t-\varepsilon)^{2}}{2 t}\right\}, \quad t>0 .
$$

Thus, as $\varepsilon \rightarrow 0$,

$$
\mathrm{P}\left(H_{2,0}^{(0)}(-\varepsilon)>1\right)=\frac{\varepsilon}{\sqrt{2 \pi}} \int_{1}^{\infty} t^{-3 / 2} \mathrm{e}^{-\varepsilon^{2} / 2 t} \mathrm{~d} t \sim \frac{2 \varepsilon}{\sqrt{2 \pi}},
$$

and, consequently,

$$
\begin{aligned}
\lim _{\varepsilon \rightarrow 0} \frac{\mathrm{P}\left(H_{\alpha, \beta}^{(u)}(-\varepsilon)>1\right)}{\mathrm{P}\left(H_{\alpha, \beta}^{(0)}(-\varepsilon)>1\right)} & =\lim _{\varepsilon \rightarrow 0} \int_{1}^{\infty} \frac{1}{2 t^{3 / 2}} \exp \left\{-\frac{(u t-\varepsilon)^{2}}{2 t}\right\} \mathrm{d} t \\
& =\int_{1}^{\infty} \frac{1}{2 t^{3 / 2}} \mathrm{e}^{-u^{2} t / 2} \mathrm{~d} t \\
& =u \int_{u}^{\infty} v^{-2} \mathrm{e}^{-v^{2} / 2} \mathrm{~d} v
\end{aligned}
$$

As a result, we have

$$
1-F_{2,0}(u)=u \int_{u}^{\infty} v^{-2} \mathrm{e}^{-v^{2} / 2} \mathrm{~d} v .
$$

This equality can be generalised to stable Lévy processes without negative jumps, i.e. $\{\alpha \in$ $(1,2), \beta=1\}$ or $\{\alpha=2, \beta=0\}$. Indeed, using Kendall's equality (see [8]) and the scaling property of stable processes, we see that $H_{\alpha, 1}^{(u)}(-\varepsilon)$ has the density

$$
u \mapsto \frac{\varepsilon}{t^{1+1 / \alpha}} g_{\alpha, 1}\left(\frac{-\varepsilon+u t}{t^{1 / \alpha}}\right) .
$$

Then, analogously to the case of the Brownian motion,

$$
1-F_{\alpha, 1}(u)=\frac{u^{1 /(\alpha-1)}}{(\alpha-1) g_{\alpha, 1}(0)} \int_{u}^{\infty} v^{-\alpha /(\alpha-1)} g_{\alpha, 1}(v) \mathrm{d} v .
$$

Unfortunately, we cannot give an explicit expression for $1-F_{\alpha, \beta}$ for a process with positive jumps. But we can describe this function via the Laplace transform of $x^{\rho-1}\left(1-F_{\alpha, \beta}\left(x^{1-1 / \alpha}\right)\right)$.

In order to prove (16), we show that $1-F_{\alpha, \beta}$ satisfies a certain integral equation. Dividing both parts of (29) by $n \mathrm{P}\left(\tau^{(0)}>n\right)$, we have

$$
\frac{\mathrm{P}\left(\tau^{(a)}>n\right)}{\mathrm{P}\left(\tau^{(0)}>n\right)}=\sum_{j=0}^{n-1} \frac{\mathrm{P}\left(\tau^{(a)}>j\right)}{\mathrm{P}\left(\tau^{(0)}>j\right)} \frac{\mathrm{P}\left(\tau^{(0)}>j\right)}{\mathrm{P}\left(\tau^{(0)}>n\right)} \mathrm{P}\left(S_{n-j}^{(0)} \geq a(n-j)\right) \frac{1}{n} .
$$


Fix any $\varepsilon \in\left(0, \frac{1}{2}\right)$. We first note that

$$
\sum_{0 \leq j \leq \varepsilon n} \frac{\mathrm{P}\left(\tau^{(a)}>j\right)}{\mathrm{P}\left(\tau^{(0)}>j\right)} \frac{\mathrm{P}\left(\tau^{(0)}>j\right)}{\mathrm{P}\left(\tau^{(0)}>n\right)} \mathrm{P}\left(S_{n-j}^{(0)} \geq a(n-j)\right) \frac{1}{n} \leq \frac{\sum_{0 \leq j \leq \varepsilon n} \mathrm{P}\left(\tau^{(0)}>j\right)}{n \mathrm{P}\left(\tau^{(0)}>n\right)} \leq C \varepsilon^{\rho}
$$

and

$$
\sum_{(1-\varepsilon) n \leq j \leq n-1} \frac{\mathrm{P}\left(\tau^{(a)}>j\right)}{\mathrm{P}\left(\tau^{(0)}>j\right)} \frac{\mathrm{P}\left(\tau^{(0)}>j\right)}{\mathrm{P}\left(\tau^{(0)}>n\right)} \mathrm{P}\left(S_{n-j}^{(0)} \geq a(n-j)\right) \frac{1}{n} \leq \frac{\mathrm{P}\left(\tau^{(0)}>n / 2\right)}{n \mathrm{P}\left(\tau^{(0)}>n\right)} \varepsilon n \leq C \varepsilon .
$$

In both bounds we have used the fact that $\mathrm{P}\left(\tau^{(0)}>j\right)$ varies regularly with index $\rho-1$.

It remains to consider the middle part of the sum on the right-hand side of (42). It is easy to see that the condition $a n / c_{n} \rightarrow u$ implies that

$$
\frac{a j}{c_{j}} \rightarrow u t^{1-1 / \alpha} \quad \text { as } a \rightarrow 0
$$

provided that $j \sim t n$. Then, in view of (40), for every $t \in(0,1)$, the following is valid. As $a \rightarrow 0$,

$$
\begin{aligned}
f_{a}(t) & :=\frac{\mathrm{P}\left(\tau^{(a)}>[t n]\right)}{\mathrm{P}\left(\tau^{(0)}>[t n]\right)} \frac{\mathrm{P}\left(\tau^{(0)}>[t n]\right)}{\mathrm{P}\left(\tau^{(0)}>n\right)} \mathrm{P}\left(S_{n-[t n]}^{(0)} \geq a(n-[t n])\right) \\
& \rightarrow\left(1-F_{\alpha, \beta}\left(u t^{1-1 / \alpha}\right)\right) t^{\rho-1} \mathrm{P}\left(Y_{\alpha, \beta}(1)>u(1-t)^{1-1 / \alpha}\right) .
\end{aligned}
$$

Thus, by dominated convergence,

$$
\begin{aligned}
\lim _{a \rightarrow 0} & \sum_{\varepsilon n<j<(1-\varepsilon) n} \frac{\mathrm{P}\left(\tau^{(a)}>j\right)}{\mathrm{P}\left(\tau^{(0)}>j\right)} \frac{\mathrm{P}\left(\tau^{(0)}>j\right)}{\mathrm{P}\left(\tau^{(0)}>n\right)} \mathrm{P}\left(S_{n-j}^{(0)} \geq a(n-j)\right) \frac{1}{n} \\
= & \int_{\varepsilon}^{1-\varepsilon}\left(1-F_{\alpha, \beta}\left(u t^{1-1 / \alpha}\right)\right) t^{\rho-1} \mathrm{P}\left(Y_{\alpha, \beta}(1)>u(1-t)^{1-1 / \alpha}\right) \mathrm{d} t .
\end{aligned}
$$

Now using monotone convergence, we obtain

$$
\begin{gathered}
\lim _{\varepsilon \rightarrow 0} \lim _{a \rightarrow 0} \sum_{\varepsilon n<j<(1-\varepsilon) n} \frac{\mathrm{P}\left(\tau^{(a)}>j\right)}{\mathrm{P}\left(\tau^{(0)}>j\right)} \frac{\mathrm{P}\left(\tau^{(0)}>j\right)}{\mathrm{P}\left(\tau^{(0)}>n\right)} \mathrm{P}\left(S_{n-j}^{(0)} \geq a(n-j)\right) \frac{1}{n} \\
=\int_{0}^{1}\left(1-F_{\alpha, \beta}\left(u t^{1-1 / \alpha}\right)\right) t^{\rho-1} \mathrm{P}\left(Y_{\alpha, \beta}(1)>u(1-t)^{1-1 / \alpha}\right) \mathrm{d} t .
\end{gathered}
$$

Combining (42)-(45), and taking into account (40), we obtain

$$
1-F_{\alpha, \beta}(u)=\int_{0}^{1}\left(1-F_{\alpha, \beta}\left(u t^{1-1 / \alpha}\right)\right) t^{\rho-1} \mathrm{P}\left(Y_{\alpha, \beta}(1)>u(1-t)^{1-1 / \alpha}\right) \mathrm{d} t .
$$

Setting

$$
G_{\alpha, \beta}(u):=1-F_{\alpha, \beta}\left(u^{1-1 / \alpha}\right) \quad \text { and } \quad \xi_{\alpha, \beta}:=\left(Y_{\alpha, \beta}(1)\right)^{\alpha /(\alpha-1)},
$$

we can rewrite (46) as follows:

$$
G_{\alpha, \beta}(u)=\int_{0}^{1} G_{\alpha, \beta}(u t) t^{\rho-1} \mathrm{P}\left(\xi_{\alpha, \beta}>u(1-t)\right) \mathrm{d} t
$$


Substituting $t=y / u$, we have

$$
G_{\alpha, \beta}(u)=u^{-\rho} \int_{0}^{u} G_{\alpha, \beta}(y) y^{\rho-1} \mathrm{P}\left(\xi_{\alpha, \beta}>u-y\right) \mathrm{d} y .
$$

Therefore, the function $Q_{\alpha, \beta}(u):=u^{\rho-1} G_{\alpha, \beta}(u)$ satisfies the equation

$$
u Q_{\alpha, \beta}(u)=\int_{0}^{u} Q_{\alpha, \beta}(y) \mathrm{P}\left(\xi_{\alpha, \beta}>u-y\right) \mathrm{d} y .
$$

Let $q_{\alpha, \beta}(\lambda)$ denote the Laplace transform of the function $Q_{\alpha, \beta}$, i.e.

$$
q_{\alpha, \beta}(\lambda)=\int_{0}^{\infty} \mathrm{e}^{-\lambda u} Q_{\alpha, \beta}(u) \mathrm{d} u, \quad \lambda>0 .
$$

Now (47) implies that

$$
\begin{aligned}
\frac{\mathrm{d}}{\mathrm{d} \lambda} q_{\alpha, \beta}(\lambda) & =-\int_{0}^{\infty} u \mathrm{e}^{-\lambda u} Q_{\alpha, \beta}(u) \mathrm{d} u \\
& =-\int_{0}^{\infty} \mathrm{e}^{-\lambda u} \int_{0}^{u} Q_{\alpha, \beta}(y) \mathrm{P}\left(\xi_{\alpha, \beta}>u-y\right) \mathrm{d} y \\
& =-\int_{0}^{\infty} \mathrm{e}^{-\lambda u} Q_{\alpha, \beta}(u) \mathrm{d} u \int_{0}^{\infty} \mathrm{e}^{-\lambda z} \mathrm{P}\left(\xi_{\alpha, \beta}>z\right) \mathrm{d} z \\
& =-q_{\alpha, \beta}(\lambda) \int_{0}^{\infty} \mathrm{e}^{-\lambda z} \mathrm{P}\left(\xi_{\alpha, \beta}>z\right) \mathrm{d} z
\end{aligned}
$$

Solving this differential equation, we see that

$$
\begin{aligned}
q_{\alpha, \beta}(\lambda) & =q_{\alpha, \beta}\left(\lambda_{0}\right) \exp \left\{-\int_{\lambda_{0}}^{\lambda} \int_{0}^{\infty} \mathrm{e}^{-\lambda z} \mathrm{P}\left(\xi_{\alpha, \beta}>z\right) \mathrm{d} z\right\} \\
& =q_{\alpha, \beta}\left(\lambda_{0}\right) \exp \left\{-\int_{0}^{\infty} \frac{\mathrm{e}^{-\lambda_{0} z}-\mathrm{e}^{-\lambda z}}{z} \mathrm{P}\left(\xi_{\alpha, \beta}>z\right) \mathrm{d} z\right\} .
\end{aligned}
$$

It follows from the definition of $\xi_{\alpha, \beta}$ that

$$
\mathrm{P}\left(\xi_{\alpha, \beta}>z\right)=\mathrm{P}\left(Y_{\alpha, \beta}(1)>z^{1-1 / \alpha}\right) \sim \frac{C}{z^{\alpha-1}} \quad \text { as } z \rightarrow \infty .
$$

This relation yields

$$
\int_{1}^{\infty} \frac{1}{z} \mathrm{P}\left(\xi_{\alpha, \beta}>z\right) \mathrm{d} z<\infty
$$

Therefore,

$$
\begin{aligned}
\int_{0}^{\infty} & \frac{\mathrm{e}^{-\lambda_{0} z}-\mathrm{e}^{-\lambda z}}{z} \mathrm{P}\left(\xi_{\alpha, \beta}>z\right) \mathrm{d} z \\
& =\int_{0}^{\infty} \frac{1-\mathrm{e}^{-\lambda z}}{z} \mathrm{P}\left(\xi_{\alpha, \beta}>z\right) \mathrm{d} z-\int_{0}^{\infty} \frac{1-\mathrm{e}^{-\lambda_{0} z}}{z} \mathrm{P}\left(\xi_{\alpha, \beta}>z\right) \mathrm{d} z
\end{aligned}
$$

Consequently,

$$
q_{\alpha, \beta}(\lambda)=C \exp \left\{-\int_{0}^{\infty} \frac{1-\mathrm{e}^{-\lambda z}}{z} \mathrm{P}\left(\xi_{\alpha, \beta}>z\right) \mathrm{d} z\right\} .
$$

To complete the proof of the theorem, it remains to note that, in view of the scaling property of $Y_{\alpha, \beta}$,

$$
\mathrm{P}\left(\xi_{\alpha, \beta}>z\right)=\mathrm{P}\left(Y_{\alpha, \beta}(1)>z^{1-1 / \alpha}\right)=\mathrm{P}\left(Y_{\alpha, \beta}(z)-z>0\right) .
$$




\section{Proof of Theorem 2}

For every $\varepsilon \in(0,1)$,

$$
\mathrm{E}\left(\tau^{(a)}\right)^{r}=\sum_{n=0}^{\infty}\left[(n+1)^{r}-n^{r}\right] \mathrm{P}\left(\tau^{(a)}>n\right)=\Sigma_{1}+\Sigma_{2}+\Sigma_{3},
$$

where

$$
\begin{aligned}
& \Sigma_{1}:=\sum_{0 \leq n \leq \varepsilon n_{a}}\left[(n+1)^{r}-n^{r}\right] \mathrm{P}\left(\tau^{(a)}>n\right), \\
& \Sigma_{2}:=\sum_{\varepsilon n_{a}<n<n_{a} / \varepsilon}\left[(n+1)^{r}-n^{r}\right] \mathrm{P}\left(\tau^{(a)}>n\right), \\
& \Sigma_{3}:=\sum_{n \geq n_{a} / \varepsilon}\left[(n+1)^{r}-n^{r}\right] \mathrm{P}\left(\tau^{(a)}>n\right) .
\end{aligned}
$$

Since $\left[(n+1)^{r}-n^{r}\right] \leq C n^{r-1}$,

$$
\Sigma_{1} \leq C \sum_{0 \leq n \leq \varepsilon n_{a}} n^{r-1} \mathrm{P}\left(\tau^{(0)}>n\right) \leq C \varepsilon^{\rho+r-1} n_{a}^{r} \mathrm{P}\left(\tau^{(0)}>n_{a}\right) .
$$

In the last step we used the fact that $\mathrm{P}\left(\tau^{(0)}>n\right)$ is regularly varying with index $\rho-1$.

Furthermore, in view of (40),

$$
\begin{aligned}
\psi_{a}(r ; x) & :=\left(\left(\left[x n_{a}\right]+1\right)^{r}-\left(\left[x n_{a}\right]\right)^{r}\right) \frac{\mathrm{P}\left(\tau^{(a)}>\left[x n_{a}\right]\right)}{n_{a}^{r-1} \mathrm{P}\left(\tau^{(0)}>n_{a}\right)} \\
& =\frac{\left(\left[x n_{a}\right]+1\right)^{r}-\left(\left[x n_{a}\right]\right)^{r}}{n_{a}^{r-1}} \frac{\mathrm{P}\left(\tau^{(a)}>\left[x n_{a}\right]\right)}{\mathrm{P}\left(\tau^{(0)}>\left[x n_{a}\right]\right)} \frac{\mathrm{P}(0)}{\mathrm{P}\left(\tau^{(0)}>n_{a}\right)} \\
& \rightarrow r x^{r-1}\left(1-F_{\alpha, \beta}\left(x^{1-1 / \alpha}\right)\right) x^{\rho-1} \quad \text { as } a \rightarrow 0 .
\end{aligned}
$$

Then, by dominated convergence,

$$
\begin{aligned}
\lim _{a \rightarrow 0} \frac{\Sigma_{2}}{n_{a}^{r} \mathrm{P}\left(\tau^{(0)}>n_{a}\right)} & =\lim _{a \rightarrow 0} \int_{\varepsilon}^{1 / \varepsilon} \psi_{a}(r ; x) \mathrm{d} x \\
& =\int_{\varepsilon}^{1 / \varepsilon} x^{r-1}\left(1-F_{\alpha, \beta}\left(x^{1-1 / \alpha}\right)\right) x^{\rho-1} \mathrm{~d} x .
\end{aligned}
$$

In view of Proposition 1,

$$
\Sigma_{3} \leq C \mathrm{E} \tau^{(a)} \sum_{n \geq n_{a} / \varepsilon} n^{r-1} \frac{V(n a)}{(n a)^{2}} .
$$

Since $V(x)$ varies regularly,

$$
\begin{aligned}
\sum_{n \geq n_{a} / \varepsilon} n^{r-1} \frac{V(n a)}{(n a)^{2}} & \sim a^{-r} \int_{a n_{a} / \varepsilon}^{\infty} x^{r-3} V(x) \mathrm{d} x \\
& \sim(\alpha-r)^{-1} \varepsilon^{\alpha-r} a^{-r}\left(a n_{a}\right)^{r-2} V\left(a n_{a}\right) \\
& \sim(\alpha-r)^{-1} \varepsilon^{\alpha-r} n_{a}^{r} \frac{V\left(a n_{a}\right)}{\left(a n_{a}\right)} \\
& \sim(\alpha-r)^{-1} \varepsilon^{\alpha-r} n_{a}^{r-1} .
\end{aligned}
$$


Here we used the relations

$$
a n_{a} \sim c_{n_{a}} \quad \text { as } a \rightarrow 0
$$

and

$$
c_{n}^{-2} V\left(c_{n}\right) \sim n^{-1} \quad \text { as } n \rightarrow \infty .
$$

Consequently,

$$
\Sigma_{3} \leq C \varepsilon^{\alpha-r} \mathrm{E} \tau^{(a)} n_{a}^{r-1} .
$$

Substituting (49)-(51) with $r=1$ into (48) with $r=1$, we have

$$
\limsup _{a \rightarrow 0} \frac{\mathrm{E} \tau^{(a)}}{n_{a} \mathrm{P}\left(\tau^{(0)}>n_{a}\right)} \leq \frac{1}{1-C \varepsilon^{\alpha-1}}\left(\int_{\varepsilon}^{1 / \varepsilon}\left(1-F_{\alpha, \beta}\left(x^{1-1 / \alpha}\right)\right) x^{\rho-1} \mathrm{~d} x+C \varepsilon^{\rho}\right) .
$$

Thus,

$$
\mathrm{E} \tau^{(a)} \leq C n_{a} \mathrm{P}\left(\tau^{(0)}>n_{a}\right) .
$$

Applying this inequality to (51) we find that

$$
\Sigma_{3} \leq C \varepsilon^{\alpha-r} n_{a}^{r} \mathrm{P}\left(\tau^{(0)}>n_{a}\right) .
$$

Combining (48)-(50) and (52), we obtain

$$
\liminf _{a \rightarrow 0} \frac{\mathrm{E}\left(\tau^{(a)}\right)^{r}}{n_{a}^{r} \mathrm{P}\left(\tau^{(0)}>n_{a}\right)} \geq \int_{\varepsilon}^{1 / \varepsilon} x^{r-1}\left(1-F_{\alpha, \beta}\left(x^{1-1 / \alpha}\right)\right) x^{\rho-1} \mathrm{~d} x
$$

and

$$
\limsup _{a \rightarrow 0} \frac{\mathrm{E}\left(\tau^{(a)}\right)^{r}}{n_{a}^{r} \mathrm{P}\left(\tau^{(0)}>n_{a}\right)} \leq \int_{\varepsilon}^{1 / \varepsilon} x^{r-1}\left(1-F_{\alpha, \beta}\left(x^{1-1 / \alpha}\right)\right) x^{\rho-1} \mathrm{~d} x+C \varepsilon^{\rho+r-1}+C \varepsilon^{\alpha-r} .
$$

The latter inequality yields

$$
\limsup _{a \rightarrow 0} \frac{\mathrm{E}\left(\tau^{(a)}\right)^{r}}{n_{a}^{r} \mathrm{P}\left(\tau^{(0)}>n_{a}\right)}<\infty
$$

Hence, letting $\varepsilon \rightarrow 0$,

$$
\lim _{a \rightarrow 0} \frac{\mathrm{E}\left(\tau^{(a)}\right)^{r}}{n_{a}^{r} \mathrm{P}\left(\tau^{(0)}>n_{a}\right)}=\int_{0}^{\infty} x^{r-1}\left(1-F_{\alpha, \beta}\left(x^{1-1 / \alpha}\right)\right) x^{\rho-1} \mathrm{~d} x .
$$

The integral $\int_{0}^{\infty} x^{r-1}\left(1-F_{\alpha, \beta}\left(x^{1-1 / \alpha}\right)\right) x^{\rho-1} \mathrm{~d} x$ is finite in view of (53). Noting now that $n_{a}^{r} \mathrm{P}\left(\tau^{(0)}>n_{a}\right)$ is regularly varying with index $-\alpha(\rho+r-1) /(\alpha-1)$, we complete the proof of the theorem.

\section{Proofs of large deviation results}

\subsection{Proof of Theorem 3}

Since $a n / c_{n} \rightarrow \infty$, there exists $N(n)$ satisfying

$$
\frac{a N(n)}{c_{n}} \rightarrow \infty \quad \text { and } \quad(n)=o(n) .
$$


We now split the sum in (29) into two parts:

$$
\begin{aligned}
& \Sigma_{1}:=\sum_{k=0}^{N(n)} \mathrm{P}\left(\tau^{(a)}>k\right) \mathrm{P}\left(S_{n-k}^{(0)}>(n-k) a\right), \\
& \Sigma_{2}:=\sum_{k=N(n)+1}^{n-1} \mathrm{P}\left(\tau^{(a)}>k\right) \mathrm{P}\left(S_{n-k}^{(0)}>(n-k) a\right) .
\end{aligned}
$$

Since

$$
\lim _{j \rightarrow \infty} \sup _{x>q_{j} c_{j}}\left|\frac{\mathrm{P}\left(S_{j}^{(0)}>x\right)}{j \mathrm{P}(X>x)}-1\right|=0
$$

for any sequence $q_{j} \uparrow \infty$, we obtain the relation

$$
\begin{aligned}
\Sigma_{1} & =(1+o(1)) n \mathrm{P}(X>n a) \sum_{k=0}^{N(n)} \mathrm{P}\left(\tau^{(a)}>k\right) \\
& =(1+o(1)) n \mathrm{P}(X>n a)\left(\mathrm{E} \tau^{(a)}-\sum_{k=N(n)+1}^{n-1} \mathrm{P}\left(\tau^{(a)}>k\right)\right) .
\end{aligned}
$$

Noting that $N(n) \gg n_{a}$ and taking into account (51), we see that

$$
\sum_{k=N(n)+1}^{n-1} \mathrm{P}\left(\tau^{(a)}>k\right)=o\left(\mathrm{E} \tau^{(a)}\right) .
$$

Combining (55) and (56), we have

$$
\Sigma_{1}=(1+o(1)) n \mathrm{E} \tau^{(a)} \mathrm{P}(X>n a) .
$$

We now turn our attention to $\Sigma_{2}$. It follows from Proposition 1 that

$$
\begin{aligned}
\Sigma_{2} & \leq \mathrm{P}\left(\tau^{(a)}>N(n)\right) \sum_{j=1}^{n} \mathrm{P}\left(S_{j}^{(0)}>a j\right) \\
& \leq C \mathrm{E} \tau^{(a)} \frac{V(a N(n))}{(a N(n))^{2}} \sum_{j=1}^{n} \mathrm{P}\left(S_{j}^{(0)}>a j\right) .
\end{aligned}
$$

Furthermore, using (32), we obtain

$$
\Sigma_{2} \leq \mathrm{E} \tau^{(a)} \frac{V(a N(n))}{(a N(n))^{2}} n^{2} \mathrm{P}(|X| \geq n a) .
$$

From the definition of $c_{n}$ and the relation $a N(n) \gg c_{n}$, we conclude that

$$
\frac{V(a N(n))}{(a N(n))^{2}}=o\left(\frac{1}{n}\right) .
$$

Moreover, $\mathrm{P}(|X| \geq n a) \leq C \mathrm{P}(X \geq n a)$ for every $X \in \mathcal{D}(\alpha, \beta)$ with $\alpha<2$ and $\beta>-1$. Then, (58) implies that

$$
\Sigma_{2}=o\left(n \mathrm{E} \tau^{(a)} \mathrm{P}(X>n a)\right) .
$$

Substituting (57) and (59) into (29) completes the proof. 


\subsection{Proof of Theorem 4}

Recall definition (24) of $\xi(a)$. Set

$$
\phi_{j}:=\mathrm{e}^{\xi(a) j} \mathrm{P}\left(\tau^{(a)}>j\right) \quad \text { and } \quad \theta_{j}:=\mathrm{e}^{\xi(a) j} \mathrm{P}\left(S_{j}^{(0)}>a j\right) .
$$

It is easily seen that

$$
\theta_{j} \leq C \text { for all } j \leq 1 / a^{2} .
$$

Furthermore, combining (21) with the relations

$$
\bar{\Phi}(x) \leq \frac{1}{x \sqrt{2 \pi}} \mathrm{e}^{-x^{2} / 2}
$$

and

we obtain

$$
\bar{\Phi}(x) \sim \frac{1}{x \sqrt{2 \pi}} \mathrm{e}^{-x^{2} / 2} \quad \text { as } x \rightarrow \infty,
$$

$$
\theta_{j} \leq \frac{C}{a \sqrt{j}} \text { for } j \leq n
$$

and

$$
\theta_{j} \sim \frac{1}{a \sqrt{2 \pi j}} \text { for } j \leq n \text { and } j a^{2} \rightarrow \infty
$$

respectively.

Multiplying both sides of (29) by $\mathrm{e}^{a^{2} n / 2}$, we see that the sequence $\phi_{j}$ satisfies the equation

$$
k \phi_{k}=\sum_{j=0}^{k-1} \phi_{j} \theta_{k-j}, \quad k \geq 1 .
$$

If $n$ satisfies the conditions of the theorem then, using (61) and (62), we have

$$
\sup _{n \geq 1} \max _{j \leq n} \theta_{j}<\infty \text {. }
$$

Consequently,

$$
\phi_{k} \leq \frac{C}{k} \sum_{j=o}^{k} \phi_{j} \leq \frac{C}{k} \sum_{j=o}^{n} \phi_{j}
$$

for all $k \leq n$. Setting $\sigma_{n}:=\sum_{j=0}^{n} \phi_{j}$, we rewrite the latter bound as

$$
\phi_{k} \leq \frac{C}{k} \sigma_{n}, \quad k \leq n .
$$

Now, applying this bound and (62) to the terms on the right-hand side of (64), we obtain, for all $k \leq n$, the bound

$$
\begin{aligned}
\phi_{k} & =\frac{1}{k} \sum_{0 \leq j<k / 2} \phi_{j} \theta_{k-j}+\frac{1}{k} \sum_{k / 2 \leq j<k} \phi_{j} \theta_{k-j} \\
& \leq \frac{C}{a k^{3 / 2}} \sum_{0 \leq j<k / 2} \phi_{j}+\frac{C \sigma_{n}}{k^{2}} \sum_{k / 2 \leq j<k} \theta_{k-j} \\
& \leq \frac{C \sigma_{n}}{a k^{3 / 2}}+\frac{C \sigma_{n}}{k^{2}} \sum_{1 \leq j<k} \frac{1}{a \sqrt{j}} \\
& \leq \frac{C \sigma_{n}}{a k^{3 / 2}} .
\end{aligned}
$$


This inequality allows us to determine the asymptotic behaviour of $\phi_{n}$. First of all we note that (63) yields

$$
\sum_{0 \leq j \leq N(n)} \phi_{j} \theta_{n-j} \sim \frac{1}{a \sqrt{2 \pi n}} \sum_{0 \leq j \leq N(n)} \phi_{j} \quad \text { as } a \rightarrow 0
$$

for every $N(n)=o(n)$. Moreover, by (65),

$$
0 \leq \sigma_{n}-\sum_{0 \leq j \leq N(n)} \phi_{j}=\sum_{N(n)<j \leq n} \phi_{j} \leq \frac{C \sigma_{n}}{a N(n)} .
$$

Therefore, choosing $N(n)$ satisfying

$$
N(n)=o(n) \quad \text { and } \quad a N^{2}(n) \rightarrow \infty,
$$

we have, as $a \rightarrow 0$,

$$
\sum_{0 \leq j \leq N(n)} \phi_{j} \theta_{n-j} \sim \frac{\sigma_{n}}{a \sqrt{2 \pi n}} .
$$

Furthermore, it follows from (62) and (65) that

$$
\begin{aligned}
\sum_{N(n)<j<n / 2} \phi_{j} \theta_{n-j} & \leq \frac{C}{a \sqrt{n}} \sum_{N(n)<j<n / 2} \phi_{j} \\
& \leq \frac{C}{a \sqrt{n}} \sum_{N(n)<j<n / 2} \frac{\sigma_{n}}{a j^{3 / 2}} \\
& \leq \frac{C \sigma_{n}}{a^{2} \sqrt{n N(n)}}
\end{aligned}
$$

and

$$
\sum_{n / 2 \leq j<n} \phi_{j} \theta_{n-j} \leq \frac{C \sigma_{n}}{a n^{3 / 2}} \sum_{j=1}^{n} \theta_{j} \leq \frac{C \sigma_{n}}{a n^{3 / 2}} \sum_{j=1}^{n} \frac{1}{a \sqrt{j}} \leq \frac{C \sigma_{n}}{a^{2} n} .
$$

Combining (67)-(69) and recalling that $a^{2} N(n) \rightarrow \infty$, we obtain

$$
\sum_{j=0}^{n-1} \phi_{j} \theta_{n-j} \sim \frac{\sigma_{n}}{a \sqrt{2 \pi n}} \quad \text { as } a \rightarrow 0 .
$$

Substituting this into (64) we have

$$
\phi_{n} \sim \frac{\sigma_{n}}{a \sqrt{2 \pi} n^{3 / 2}} \quad \text { as } a \rightarrow 0 .
$$

To complete the proof of the theorem, it remains to find the asymptotic behaviour of $\sigma_{n}$. First of all, (66) implies that the bounds

$$
\sum_{j \leq 1 / \varepsilon a^{2}} \phi_{j} \leq \sigma_{n} \leq(1-C \sqrt{\varepsilon})^{-1} \sum_{j \leq 1 / \varepsilon a^{2}} \phi_{j}
$$


are valid for all sufficiently small values of $\varepsilon$. Applying Theorem 1 and recalling that $\mathrm{P}\left(\tau^{(0)}>j\right)$ is regularly varying with index $-\frac{1}{2}$, we see that

$$
\begin{aligned}
\lim _{a \rightarrow 0} \frac{\phi_{\left[x a^{-2}\right]} \mathrm{P}\left(\tau^{(0)}>a^{-2}\right)}{} & =\lim _{a \rightarrow 0} \frac{\mathrm{e}^{\left[x a^{-2}\right] \xi(a)} \mathrm{P}\left(\tau^{(a)}>\left[x a^{-2}\right]\right)}{\mathrm{P}\left(\tau^{(0)}>\left[x a^{-2}\right]\right)} \frac{\mathrm{P}\left(\tau^{(0)}>\left[x a^{-2}\right]\right)}{\mathrm{P}\left(\tau^{(0)}>a^{-2}\right)} \\
& =\mathrm{e}^{x / 2}\left(1-F_{2,0}(\sqrt{x})\right) \frac{1}{\sqrt{x}}
\end{aligned}
$$

for every $x>0$. Thus, by dominated convergence,

$$
\begin{aligned}
\lim _{a \rightarrow 0} \frac{\sum_{j \leq 1 / \varepsilon a^{2}} \phi_{j}}{a^{-2} \mathrm{P}\left(\tau^{(0)}>a^{-2}\right)} & =\int_{0}^{1 / \varepsilon} \frac{\mathrm{e}^{x / 2}}{\sqrt{x}}\left(1-F_{2,0}(\sqrt{x})\right) \mathrm{d} x \\
& =: I(\varepsilon) .
\end{aligned}
$$

Using (41), we have

$$
\begin{aligned}
I(\varepsilon) & =\int_{0}^{1 / \varepsilon} \mathrm{e}^{x / 2} \int_{\sqrt{x}}^{\infty} v^{-2} \mathrm{e}^{-v^{2} / 2} \mathrm{~d} v \mathrm{~d} x \\
& =\int_{0}^{\infty} \mathrm{e}^{x / 2} \int_{\sqrt{x}}^{\infty} v^{-2} \mathrm{e}^{-v^{2} / 2} \mathrm{~d} v \mathrm{~d} x-\int_{1 / \varepsilon}^{\infty} \mathrm{e}^{x / 2} \int_{\sqrt{x}}^{\infty} v^{-2} \mathrm{e}^{-v^{2} / 2} \mathrm{~d} v \mathrm{~d} x .
\end{aligned}
$$

Noting that

$$
\int_{\sqrt{x}}^{\infty} v^{-2} \mathrm{e}^{-v^{2} / 2} \mathrm{~d} v \leq \frac{\mathrm{e}^{-x / 2}}{x^{3 / 2}}
$$

we have

$$
0 \leq \int_{0}^{\infty} \mathrm{e}^{x / 2} \int_{\sqrt{x}}^{\infty} v^{-2} \mathrm{e}^{-v^{2} / 2} \mathrm{~d} v \mathrm{~d} x-I(\varepsilon) \leq \sqrt{\varepsilon}
$$

Changing the order of integration and substituting $v^{2} / 2=u$, we have

$$
\begin{aligned}
\int_{0}^{\infty} \mathrm{e}^{x / 2} \int_{\sqrt{x}}^{\infty} v^{-2} \mathrm{e}^{-v^{2} / 2} \mathrm{~d} v \mathrm{~d} x & =\int_{0}^{\infty} v^{-2} \mathrm{e}^{-v^{2} / 2} \int_{0}^{v^{2}} \mathrm{e}^{x / 2} \mathrm{~d} x \mathrm{~d} v \\
& =2 \int_{0}^{\infty} v^{-2} \mathrm{e}^{-v^{2} / 2}\left(1-\mathrm{e}^{-v^{2} / 2}\right) \mathrm{d} v \\
& =\frac{1}{\sqrt{2}} \int_{0}^{\infty} u^{-3 / 2}\left(1-\mathrm{e}^{-u}\right) \mathrm{d} u
\end{aligned}
$$

Integrating now by parts we obtain

$$
\int_{0}^{\infty} u^{-3 / 2}\left(1-\mathrm{e}^{-u}\right) \mathrm{d} u=2 \int_{0}^{\infty} u^{-1 / 2} \mathrm{e}^{-u} \mathrm{~d} u=2 \Gamma\left(\frac{1}{2}\right)=2 \sqrt{\pi} .
$$

As a result, we have the bounds

$$
\sqrt{2 \pi}-\sqrt{\varepsilon} \leq I(\varepsilon) \leq \sqrt{2 \pi}
$$

Substituting (72) and (73) into (71), we obtain

$$
\sqrt{2 \pi}-\sqrt{\varepsilon} \leq \liminf _{a \rightarrow 0} \frac{\sigma_{n}}{a^{-2} \mathrm{P}\left(\tau^{(0)}>a^{-2}\right)} \leq \limsup _{a \rightarrow 0} \frac{\sigma_{n}}{a^{-2} \mathrm{P}\left(\tau^{(0)}>a^{-2}\right)} \leq \frac{\sqrt{2 \pi}}{1-C \sqrt{\varepsilon}} .
$$


Since $\varepsilon$ can be chosen arbitrarily small,

$$
\sigma_{n} \sim \sqrt{2 \pi} a^{-2} \mathrm{P}\left(\tau^{(0)}>a^{-2}\right) .
$$

Combining (70) and (74), and recalling definition (60) of $\phi_{n}$, we have

$$
\mathrm{P}\left(\tau^{(a)}>n\right) \sim a^{-3} n^{-3 / 2} \mathrm{e}^{-\xi(a) n} \mathrm{P}\left(\tau^{(0)}>a^{-2}\right) .
$$

Furthermore, it follows from (54) that

$$
\mathrm{E} \tau^{(a)} \sim a^{-2} \mathrm{P}\left(\tau^{(0)}>a^{-2}\right) \int_{0}^{\infty}\left(1-F_{2,0}(\sqrt{x})\right) x^{-1 / 2} \mathrm{~d} x .
$$

Substituting $\sqrt{x}=y$ and using (41), we obtain

$$
\begin{aligned}
\int_{0}^{\infty}\left(1-F_{2,0}(\sqrt{x})\right) x^{-1 / 2} \mathrm{~d} x & =2 \int_{0}^{\infty}\left(1-F_{2,0}(y)\right) \mathrm{d} y \\
& =2 \int_{0}^{\infty} y\left(\int_{y}^{\infty} v^{-2} \mathrm{e}^{-v^{2} / 2} \mathrm{~d} v\right) \mathrm{d} y \\
& =2 \int_{0}^{\infty} v^{-2} \mathrm{e}^{-v^{2} / 2}\left(\int_{0}^{v} y \mathrm{~d} y\right) \mathrm{d} v \\
& =\int_{0}^{\infty} \mathrm{e}^{-v^{2} / 2} \mathrm{~d} v \\
& =\sqrt{\frac{\pi}{2}}
\end{aligned}
$$

As a result, we have

$$
a^{-2} \mathrm{P}\left(\tau^{(0)}>a^{-2}\right) \sim \sqrt{\frac{2}{\pi}} \mathrm{E} \tau^{(a)} .
$$

Combining (75) and (76), and noting that

$$
\frac{1}{a \sqrt{2 \pi n}} \mathrm{e}^{-\xi(a) n} \sim \bar{\Phi}(a \sqrt{n}) \exp \left\{n a^{3} \lambda_{m}(a)\right\},
$$

completes the proof.

\subsection{Proof of Theorem 5}

It is easy to see that there exist a constant $C$ and a regularly varying function $N(a)$ such that

$$
\lim _{a \rightarrow 0} \frac{N(a)}{a^{-2} \log a^{-2}}=(p-2)^{1 / 2}
$$

and

$$
\sup _{n \leq N(a)} \frac{n \mathrm{P}(X \geq n a+\sqrt{n})}{\bar{\Phi}(a \sqrt{n})} \leq C \quad \text { and } \quad \sup _{n \geq N(a)} \frac{\bar{\Phi}(a \sqrt{n})}{n \mathrm{P}(X \geq n a+\sqrt{n})} \leq C .
$$

We now split the right-hand side of (10) into the product of two exponentials:

$$
\begin{aligned}
\exp \left\{\sum_{n=1}^{\infty} \frac{z^{n}}{n} \mathrm{P}\left(S_{n}^{(a)}>0\right)\right\} & =\exp \left\{\sum_{n=1}^{N(a)} \frac{z^{n}}{n} \mathrm{P}\left(S_{n}^{(a)}>0\right)\right\} \exp \left\{\sum_{n=N(a)+1}^{\infty} \frac{z^{n}}{n} \mathrm{P}\left(S_{n}^{(a)}>0\right)\right\} \\
& =:\left(\sum_{n=0}^{\infty} \psi_{1, n} z^{n}\right)\left(1+\sum_{n=N(a)+1}^{\infty} \psi_{2, n} z^{n}\right) .
\end{aligned}
$$


Therefore,

$$
\mathrm{P}\left(\tau^{(a)}>n\right)=\psi_{1, n}+\sum_{k=N(a)+1}^{n} \psi_{1, n-k} \psi_{2, k}, \quad n \geq 1 .
$$

We first want to find the asymptotic behaviour of $\psi_{2, n}$. We start by noting that

$$
\psi_{2, n}=\sum_{j=1}^{\infty} \frac{1}{j !} q_{n}^{* j}, \quad n>N(a)
$$

where $\left\{q_{n}^{* j}, n \geq 1\right\}$ is the $j$ th convolution of $\left\{n^{-1} \mathrm{P}\left(S_{n}^{(a)}>0\right) \mathbf{1}\{n>N(a)\}, n \geq 1\right\}$. It follows from the second inequality in (78) that

$$
\begin{aligned}
q_{n}^{* 2} & =\sum_{k=N(a)+1}^{n-N-1} \frac{1}{k} \mathrm{P}\left(S_{k}^{(a)}>0\right) \frac{1}{n-k} \mathrm{P}\left(S_{n-k}^{(a)}>0\right) \\
& \leq C \sum_{k=N(a)+1}^{n-N-1} \mathrm{P}(X \geq a k) \mathrm{P}(X \geq a(n-k)) \\
& \leq C \mathrm{P}\left(X \geq \frac{a n}{2}\right) \sum_{N(a)+1} \mathrm{P}(X \geq a k) \\
& \leq C \mathrm{P}(X \geq a n) \int_{N(a)}^{\infty} \mathrm{P}(X \geq a y) \mathrm{d} y
\end{aligned}
$$

Since $\mathrm{P}(X \geq y)$ is regularly varying, we have

$$
\int_{N(a)}^{\infty} \mathrm{P}(X \geq a y) \mathrm{d} y=\frac{1}{a} \int_{a N(a)}^{\infty} \mathrm{P}(X \geq y) \mathrm{d} y \leq C N(a) \mathrm{P}(X \geq a N(a)) .
$$

From this bound and (77) we obtain

$$
q_{n}^{* 2} \leq G(a) \mathrm{P}(X \geq a n),
$$

where $G$ is regularly varying with index $p-2>0$. Then, by induction,

$$
q_{n}^{* j} \leq G(a) \mathrm{P}(X \geq a n) \quad \text { for all } j \geq 2 .
$$

Combining (80) and (81), and using (23) and (78), we obtain the bound

$$
\begin{aligned}
\psi_{2, n} & =\mathrm{P}\left(S_{n}^{(a)}>0\right)+\sum_{j=2}^{\infty} q_{n}^{* j} \\
& \leq C\left(\frac{1}{n} \bar{\Phi}(a \sqrt{n})+\mathrm{P}(X \geq a n)+G(a) \mathrm{P}(X \geq a n)\right) \\
& \leq C \mathrm{P}(X \geq a n)
\end{aligned}
$$

and, for $n \geq C a^{-2} \log a^{-2}$ with some $C>(p-2)^{1 / 2}$, the relation

$$
\psi_{2, n}=\mathrm{P}\left(S_{n}^{(a)}>0\right)+O(G(a) \mathrm{P}(X \geq a n)) \sim \frac{1}{n} \bar{\Phi}(a \sqrt{n})+\mathrm{P}(X \geq a n) \sim \mathrm{P}(X \geq a n) .
$$


In the last step we have used the fact that $\bar{\Phi}(a \sqrt{n})=o(\mathrm{P}(X \geq a n))$ for $n \geq C a^{-2} \log a^{-2}$, $C>(p-2)^{1 / 2}$.

From the first inequality in (78) and (23), which is valid under condition (26), we conclude that

$$
\mathrm{P}\left(S_{n}^{(a)}>0\right) \leq C \bar{\Phi}(a \sqrt{n})
$$

for all $n \leq N(a)$. Using arguments from the proof of Theorem 4, we see that

$$
\psi_{1, k} \leq \frac{C}{k} \bar{\Phi}(a \sqrt{k}), \quad k \geq 1
$$

Combining (82) and (84), and applying the second inequality in (78), we obtain

$$
\begin{aligned}
\sum_{k=N(a)}^{n-N(a)} \psi_{1, n-k} \psi_{2, k} & \leq C \sum_{k=N(a)}^{n-N(a)} \frac{1}{n-k} \bar{\Phi}(a \sqrt{n-k}) \mathrm{P}(X \geq a k) \\
& \leq C \sum_{k=N(a)}^{n-N(a)} \mathrm{P}(X \geq a(n-k)) \mathrm{P}(X \geq a k)
\end{aligned}
$$

In the derivation of (81) we showed that the sum in the last line is bounded by $G(a) \mathrm{P}(X \geq a n)$. Hence,

$$
\sum_{k=N(a)}^{n-N(a)} \psi_{1, n-k} \psi_{2, k}=O(G(a) \mathrm{P}(X \geq a n)) .
$$

It follows from (10) and the definition of $\left\{\psi_{1, n}, n \geq 1\right\}$ that $\psi_{1, k}=\mathrm{P}\left(\tau^{(a)}>k\right)$ for all $k \leq N(a)$. Consequently,

$$
\begin{aligned}
\sum_{k=n-N(a)+1}^{n} \psi_{1, n-k} \psi_{2, k} & =\sum_{k=0}^{N(a)-1} \mathrm{P}\left(\tau^{(a)}>k\right) \psi_{2, n-k} \\
& =\sum_{k=0}^{\tilde{N}(a)-1} \mathrm{P}\left(\tau^{(a)}>k\right) \psi_{2, n-k}+\sum_{k=\tilde{N}(a)}^{N(a)-1} \mathrm{P}\left(\tau^{(a)}>k\right) \psi_{2, n-k},
\end{aligned}
$$

where $\tilde{N}(a)$ is such that $a^{-2} \ll \tilde{N}(a) \ll a^{-2} \log a^{-2}$. Applying (82) to the fist sum and (83) to the second sum, we obtain

$$
\begin{aligned}
\sum_{k=n-N(a)+1}^{n} \psi_{1, n-k} \psi_{2, k}= & (1+o(1)) \mathrm{P}(X \geq a n) \sum_{k=0}^{\tilde{N}(a)-1} \mathrm{P}\left(\tau^{(a)}>k\right) \\
& +O\left(\mathrm{P}(X \geq a n) \sum_{k=\tilde{N}(a)}^{N(a)-1} \mathrm{P}\left(\tau^{(a)}>k\right)\right) .
\end{aligned}
$$

Note that (51) implies that

$$
\sum_{k=\tilde{N}(a)}^{\infty} \mathrm{P}\left(\tau^{(a)}>k\right)=o\left(\mathrm{E} \tau^{(a)}\right)
$$


Hence, we finally obtain

$$
\sum_{k=n-N(a)+1}^{n} \psi_{1, n-k} \psi_{2, k} \sim \mathrm{E} \tau^{(a)} \mathrm{P}(X \geq a n) .
$$

Combining (79), (85), and (86), we have

$$
\mathrm{P}\left(\tau^{(a)}>n\right)=(1+o(1)) \mathrm{E} \tau^{(a)} \mathrm{P}(X \geq a n)+\psi_{1, n} .
$$

In order to complete the proof, it remains to apply (84) and to note that

$$
n^{-1} \bar{\Phi}(a \sqrt{n})=o(\mathrm{P}(X \geq a n)) .
$$

\section{Acknowledgement}

The author would like to thank Klaus Fleischmann for remarks which led to a better presentation of the results.

\section{References}

[1] Bertoin, J. (1996). Lévy Processes. Cambridge University Press.

[2] Bertoin, J. And Doney, R. A. (1996). Some asymptotic results for transient random walks. Adv. Appl. Prob. 28, 207-226.

[3] Doney, R. A. (1985). Conditional limit theorems for asymptotically stable random walks. Z. Wahrscheinlichkeitsth. 70, 351-360.

[4] Doney, R. A. (1989). On the asymptotic behaviour of first passage times for transient random walk. Prob. Theory Relat. Fields 81, 239-246.

[5] Embrechts, P. And Hawkes, J. (1982). A limit theorem for the tails of discrete infinitely divisible laws with application to fluctuation theory. J. Austral. Math. Soc. A 32, 412-422.

[6] Feller, W. (1971). An Introduction to Probability Theory and Its Applications. Vol. 2, 2nd edn. John Wiley, New York.

[7] Gut, A. (1974). On the moments and limit distributions of some first passage times. Ann. Prob. 2, $277-308$.

[8] KeILson, J. (1963). The first passage time density for homogeneous skip-free walks on the continuum. Ann. Math. Statist. 34, 1003-1011.

[9] Lotov, V. I. (2006). On the mean value of the ladder epoch for random walks with small drift. Izv. Math. 70, 1225-1232.

[10] Nagaev, A. V. (1969). Limit theorems for large deviations where Cramér's conditions are violated. Izv. Akad. Nauk UzSSR Ser. Fiz.-Mat. Nauk. 6, 17-22 (in Russian).

[11] Nagaev, S. V. (1965). Some limit theorems for large deviations. Theory Prob. Appl. 10, 214-235.

[12] Nagaev, S. V. (1979). Large deviations of sums of independent random variables. Ann. Prob. 7, 745-789.

[13] Osipov, L. V. (1972). On probabilities of large deviations for sums of independent random variables. Theory Prob. Appl. 17, 309-331.

[14] Petrov, V. V. (1975). Sums of Independent Random Variables. Springer, New York.

[15] Rogozin, B. A. (1971). On the distribution of the first ladder moment and height and fluctuations of a random walk. Theory Prob. Appl. 16, 575-595.

[16] RozovskǏ̌, L. V. (1989). Probabilities of large deviations of sums of independent random variables with a common distribution function that belongs to the domain of attraction of the normal law. Theory Prob. Appl. 34, 625-644.

[17] Spitzer, F. (2001). Principles of Random Walks. Springer, Berlin.

[18] Zolotarev, V. M. (1957). Mellin-Stieltjes transform in probability theory. Theory Prob. Appl. 2, 433-460. 